\title{
ABSTRACTS SASOP Congress 2014
}

\section{3 - 7 September 2014 \\ Southern Sun Elangeni and Maharani Hotel, Durban, South Africa}

S Afr J Psych 2014;20(3):96-128. DOI:10.7196/SAJP.664

\section{ORAL PRESENTATIONS}

\author{
DSM-5: What is up? A critical review \\ A Abdelrahman \\ Faculty of Medicine, University of Khartoum, Sudan \\ a_abdelrahman53@hotmail.com
}

The objective of this presentation is to shed light on the Diagnostic and Statistical Manual of Mental Disorders, 5th edition (DSM-5) recently issued by the American Association of Psychiatrists. Many clinicians voiced different views about this document. The document will be compared with its previous edition, DSM-IV. Some of the comments and concerns of psychiatrists and other mental health professionals will be highlighted. As any genuine work would have advantages and disadvantages, this presentation will be an endeavour to show the most important components of each case. It will also mention the most important similarities and differences of this edition with the last edition of the International Classification of Diseases (ICD-10).

How can we make private healthcare more efficient and sustainable?

\section{$\underline{\text { E Allers }}$}

Psychiatrist in private practice, Director PHI Psychiatric Management; ex-officio board member PsychMg

kopshop@global.co.za

The fee-for-service (FFS) model has been the main model of reimbursement in the private sector in South Africa. This model has been heavily criticised by the Minister of Health, the Department of Health, the medical schemes, actuaries and others for its inefficiencies. The drive to introduce a National Health Insurance is gaining momentum, against all warnings that the cost will be enormous. This is of concern as this poses a challenge to the psychiatrist in private practice. Medical inflation has annually been 3\% above the consumer price index (CPI). On a cumulative basis this relates to an exponential increase in cost for those who utilise the services. There would be a critical point at which such services would be unaffordable for patients as this eventually also means an increase in the monthly medical scheme contribution. This presentation will focus on alternatives to the FFS model.

Prefrontal cortical thinning in first-episode schizophrenia and its clinical correlates

$\underline{\text { L Asmal, }}{ }^{*}$ S du Plessis, B Chiliza, A Goosen, R Emsley
Department of Psychiatry, Stellenbosch University, Cape Town, South Africa

*laila@sun.ac.za

Background. Prefrontal grey matter deficits in schizophrenia are frequently, but not consistently, found on MRI scanning. The functional diversity of the prefrontal cortex suggests that abnormalities in the region may in part explain the difficulties in cognitive and emotional integration that characterise the clinical manifestation of schizophrenia. Here, we compare prefrontal cortical thickness between 93 patients with minimally treated first-episode schizophrenia (FES) and 92 matched healthy controls (HC). We then examine correlations between prefrontal cortical thickness and clinical symptoms scores.

Method. T1-weighted scans were processed and analysed using Freesurfer stable release version 5.1. We conducted $t$-tests corrected for multiple comparisons to compare cortical thickness differences between patients and controls. Pairwise correlations were performed between left and right hemisphere prefrontal composite and clinical measures. Results. We found a reduction in prefrontal composite thickness bilaterally as well as in the left medial orbitofrontal cortex, left frontal pole, left superior frontal gyrus, the right dorsolateral prefrontal cortex, right lateral orbitofrontal cortex and right superior frontal gyrus. We found a positive correlation between PANSS positive factor domain scores and right hemisphere prefrontal composite thickness, higher PANSS G12 Insight item score and a reduction in bilateral prefrontal composite thickness, and a negative correlation between the BIS total score and bilateral prefrontal composite thickness.

Conclusion. In this, the largest known study to specifically examine prefrontal cortical thickness and its clinical correlates in schizophrenia, we found evidence of reduced bilateral prefrontal cortical thickness as well as reduced thickness in several prefrontal cortical regions. Key prefrontal cortical deficits may be crucial to the pathogenesis of positive symptoms and insight early in schizophrenia. Longitudinal studies are needed to identify the effect of disease progression on cortical thickness and its relationship with clinical symptoms.

Impact of care environment on anxiety, depression and suicidality among orphaned adolescents in western Kenya L Atwoli

Moi University School of Medicine, Eldoret, Kenya

lukoye.atwoli@mu.ac.ke 
Background. The HIV pandemic, poverty and multiple conflicts have left in their wake a large number of orphaned and separated children in Kenya. No study has comprehensively assessed mental health outcomes in this population.

Objective. To determine the impact of care environment on anxiety, depression and suicidality in this cohort.

Method. The Orphaned and Separated Children's Assessment Related to their Physical and Mental Health study is a longitudinal project that followed 1565 adolescents in different environments (households, charitable children's institutions (CCIs) and street children) over a period of 5 years. Anxiety was assessed using the Revised Child Manifest Anxiety Scale (R-CMAS), while depression and suicidality were assessed using the Child Depression Inventory (CDI).

Results. Of the 1565 adolescents, 55\% were male, and the mean age was 13.8 years. The prevalence of depression was $13.8 \%$, while $18.5 \%$ had either suicidal ideation (14.6\%) or intent (3.9\%). Adjusting for age, sex, orphan status (single or double) and HIV status, street children had four times greater odds of having anxiety, while those in CCIs had lower odds (odds ratio (OR) 0.59) compared with those living with relatives in households. Children in CCIs also had significantly lower odds of depression (OR 0.22 ) and suicidality (OR 0.64) compared with those in households.

Conclusion. Compared with children in households, street children suffer more anxiety, while children in CCIs suffer less anxiety, depression and suicidality. Interventions are required to address the comparatively higher levels of anxiety, depression and suicidality among orphans living in households, compared with those in CCIs.

HIV prevalence and sociodemographic characteristics of patients in a forensic unit at Sterkfontein Psychiatric Hospital, South Africa

M G Beke, ${ }^{\star}$ D C J Hoffman

Division of Psychiatry, University of the Witwatersand, Johannesburg, South Africa

*mgbeke@gmail.com

Background. HIV/AIDS remains a formidable health condition and South Africa (SA) continues to be the epicentre of the pandemic. The relationship between HIV and psychiatric disorders is established. Patients in mental healthcare institutions experience a higher burden of the condition relative to the general population. Although the prevalence in the general population is well known over the years, the magnitude and distribution of HIV/AIDS in mental healthcare patients is not consistently studied and little is known about the subgroup of forensic patients in South Africa. The objective is to determine the prevalence of HIV and describe the sociodemographic characteristics of patients in a forensic unit at Sterkfontein Psychiatric Hospital, SA.

Method. Data were extracted retrospectively from state patients records on sociodemographic characteristics and health status, including HIV status, using a standardised data collection tool. The study population was all adults admitted at the forensic unit at Sterkfontein Hospital from 1 January 2007 to 31 December 2011 in terms of section 42 of the Mental Health Act 17 of 2002.

Results. Males made up $92 \%$ of the study population of 137 subjects, with a large number of people being single (84.7\%), unemployed (89.8\%) and aged between 33 and 39 years (33\%). There were two major Axis 1 diagnoses: schizophrenia (37\%) and psychosis secondary to general medical condition (GMC) (26\%). Axis II diagnosis was made in 67 patients; the conditions were mainly intellectual disability and antisocial personality disorder, both accounting for $97 \%$ of the diagnoses. Crimes committed were mainly rape (34\%), murder (30\%) and assault/grievous bodily harm (21\%), accounting for over $85 \%$ of offences. HIV testing was done in $124(89 \%)$ of the patients and HIV seroprevalence proportion was $21.8 \%$. The highest prevalence of $44.5 \%$ was found in patients with psychosis secondary to GMC and with schizophrenia at $22 \%$. However, there is no statistically significant association between HIV status and Axis 1 diagnosis. There was no statistically significant association between HIV status and sex, age, marital status, Axis II diagnosis, disability grant and employment status at $p<0.05$.

Conclusion. HIV seroprevalence is higher in the forensic unit than the general population. Efforts should be made to make an early diagnosis and manage the condition appropriately. There is no clinical or sociodemographic factor associated with HIV status. Furthermore, HIV tests were not done for $9 \%$ of the patients. Policies and procedures should be formalised for routine diagnostic testing of all patients. Efforts should also be instituted to prevent transmission within the institution. Highly active antiretroviral therapy (HAART) should be offered to all eligible patients.

\section{Long-acting injectable antipsychotic in first-episode schizophrenia: The EONKCS Study}

$\underline{\text {B Chiliza }_{2}}{ }^{*}$ L Asmal, R Emsley

Department of Psychiatry, Stellenbosch University, Cape Town,

South Africa

*bonga@sun.ac.za

Background/Objective. Depot antipsychotics were developed in the 1960s to address the adherence problem in schizophrenia. It can be argued that the greatest benefits of depot antipsychotics would be observed in the earlier phase of illness, in line with public health principles of early intervention and prevention of accruing morbidity. Our objective was to assess the feasibility and effectiveness of depot antipsychotic (flupenthixol decanoate) combined with an assertive monitoring programme (AMP) in first-episode schizophrenia.

Method. This was a prospective, non-comparative, longitudinal study conducted over 12 months assessing patient acceptance, adherence, outcome in domains of psychopathology, functionality and quality of life, and tolerability.

Results. Of 207 participants, 149 (72\%) completed 12 months' treatment. Acceptance of, and adherence was good. Treatment response was achieved by 170 (82\%) participants and remission by $124(60 \%)$. Thirtythree (19\%) responders relapsed and $10(5 \%)$ participants met a priori criteria for treatment resistance. Treatment was generally well tolerated.

Conclusion. Combination of depot antipsychotic with an AMP may be an effective and safe intervention in early phases of schizophrenia, and may be particularly suitable for resource-constrained settings.

\author{
Performance of the geriatric depression scale in an elderly \\ residential sample in Durban \\ LChipps, ${ }^{1 \star}$ S Ramlall ${ }^{2}$
}




\section{ABSTRACTS}

${ }^{1}$ University of the Western Cape, Bellville, South Africa

${ }^{2}$ University of KwaZulu-Natal, Durban, South Africa

*chippsj@gmail.com

Background. The point prevalence of depression in the elderly in the community varies from $10 \%$ to $20 \%$ with significant comorbidity with medical disorders and dementia. Depression carries significant morbidity and suicide risk. Screening the elderly for depression requires valid screening tools. The Geriatric Depression Scale (GDS 30) was specifically created and validated as a self-report tool for the elderly and has a sensitivity of $84 \%$ and a specificity of $95 \%$ at a cut-off score of $\geq 11$. Method. In a cross-sectional study conducted in an old-age facility, 302 elderly aged $\geq 60$ years were screened for subjective and objective cognitive impairment. The Subjective Memory Complaint, a single domain screening measure assessing memory through a 'yes/no' response to the question: 'Are you experiencing any difficulty with your memory;' and the Mini-Mental State Examination (MMSE) were used respectively. Depression was screened for using the GDS 30. A total of 140 of the elderly who were screened were subsequently clinically assessed for a diagnosis of depression, mild cognitive impairment and dementia.

Results. A total of 301 participants completed the GDS 30. The average GDS score was 8.6. The total GDS score was significantly associated with race $(p=0.021)$ but not age groups $(p=0.829)$, gender $(p=0.051)$ or level of education ( $p=0.099)$.Using the 30 and 15 item GDS and standard cut-off scores of $>11$ and $>5$, a total of $100(33.2 \%)$ and $95(31.6 \%)$ participants screened positive for depression, respectively. The presence of depression ( $>11$ score in GDS 30 ) was not significantly associated with race $(p=0.08)$, age $(p=0.253)$, gender $(p=0.127)$ or education $(p=0.486)$. There was a good correlation between the GDS $30 \mathrm{v}$. GDS $15(r=0.93)$. There was poor correlation between GDS and MMSE scores $(r=0.145)$. Thirteen cases $(9.3 \%)$ of major depression (current) were identified; 25 (17.9\%) participants had a history of depression. There was not a significant association between depression and cognitive impairment (dementia or mild cognitive impairment).The sensitivity and specificity of the GDS for major depression was $61.5 \%(31.6 \%-86 \%)$ and $67.2 \%(58.9 \%-$ $75.4 \%$ ), respectively. However, there was a significant association between being depressed (>11) and having an SMC, with $64 \%$ people who were depressed reporting an SMC, and $45.7 \%$ of participants with SMC being depressed on the GDS (Fisher exact $\chi^{2}=9.9, p=0.006$ ).

Conclusion. Our data on the GDS support the use of both the GDS 30 and 15 in our local population without significant influence on its performance due to race, age, gender, level of education and MMSE scores.

\section{Forensic sleep neuropsychiatry - experiences from the UK} I Ebrahim

The London Sleep Centre, London, UK

Irshaad.Ebrahim@londonsleepcentre.com

The area of forensic sleep medicine/neuropsychiatry has undergone rapid development over the past decade. Early high-profile cases in North America $(R v$. Kenneth Parks) stimulated an increase in clinical and medicolegal interest in the area. There have been significant developments in our understanding of automatism, and this has increased debate between the medical and legal community on the nature of automatism and the not-guilty-by-reason-of-insanity (NGRI) verdict. This talk will cover historical aspects of the NGRI verdict, outline the legal and forensic views on the subject through case reports, provide guidance on the clinical and forensic assessment of patients who are charged with sleeprelated injury/offences and highlight some current controversies.

Changes in brain regions associated with food-intake regulation, body mass and metabolic profiles during acute antipsychotic treatment in first-episode schizophrenia

$\underline{\text { R Emsley, }},{ }^{1 *}$ L Asmal, ${ }^{1}$ B Chiliza, ${ }^{1}$ S du Plessis, ${ }^{1}{ }^{1}$ Carr, ${ }^{2}$ M Kidd, ${ }^{3}$

A K Malhotra, ${ }^{4}$ M Vink, ${ }^{5}$ R S Kahn ${ }^{5}$

${ }^{1}$ Department of Psychiatry, Stellenbosch University, Cape Town, South Africa

${ }^{2}$ Division of Neurology, Stellenbosch University

${ }^{3}$ Centre for Statistical Consultation, Stellenbosch University

${ }^{4}$ Division of Psychiatric Research, The Zucker Hillside Hospital, Glen Oaks, New York, USA

${ }^{5}$ Department of Psychiatry, University Medical Centre Utrecht, The Netherlands

*rae@sun.ac.za

Background. We investigated whether morphological brain changes occurred in brain regions associated with body-weight homeostasis during acute antipsychotic treatment, and if so, whether they were related to changes in body mass and metabolic profile.

Method. Twenty-two antipsychotic-naive patients with first-episode schizophrenia received either risperidone long-acting injection or flupenthixol decanoate over 13 weeks and were compared by structural magnetic resonance imaging (MRI) with 23 matched healthy volunteers at weeks 0,4 and 13. Images were reconstructed using Freesurfer fully-automated whole-brain segmentation including a longitudinal processing stream. The ventral diencephalon and prefrontal cortex were selected to represent the homeostatic and hedonic food intake regulatory systems respectively. Body mass was measured at weeks 0,7 and 13 , and fasting glucose and lipid profiles at weeks 0 and 13 .

Results. Linear mixed effect models indicated significant reductions in ventral diencephalic volume, which were strongly correlated bilaterally with body mass increase and high-density lipoprotein-cholesterol reductions, and unilaterally with blood glucose elevation. There were no significant changes in prefrontal cortical thickness.

Conclusion. These findings implicate the ventral diencephalon, of which the hypothalamus is the main component, in the acute adipogenic and dyslipidaemic effects of antipsychotic medication.

SASSA and psychiatry: A retrospective analysis of 100 disability grant applications from rural KwaZulu-Natal L Eriksson $^{\star}$, H Sorour

GJ Crookes Hospital, Scottburgh, KwaZulu-Natal, South Africa *lennarte@iafrica.com

Background. This presentation will highlight: (i) the clinical demands of mental health professionals in aiding and supporting citizens who are disabled by a serious and persistent mental illness or other neuropsychiatric disease or disorder; (ii) the current South African Social Security Agency (SASSA) criteria for the approval or repudiation of a disability grant application for persons with a neuropsychiatric disease 
or disorder; (iii) the current demands being made on the SASSA medical officer to approve or repudiate a disability grant application on the grounds of mental illness; (iv) the current format and content of the SASSA reports on which this study is based; and $(v)$ an analysis of 100 disability grant applications made to two rural KwaZulu-Natal facilities. Method/results. One hundred SASSA clinical reports will be analysed for demographic data, clinical diagnosis and degree of mental disability. Conclusion. The processing of SASSA disability grant applications in respect of mentally ill applicants is in need of both clinical and administrative review. The analysis presented will demonstrate the wide range of persons with neuropsychiatric diseases and disorders who make application to receive a SASSA disability grant.

\section{Definition of psychosis and classification and diagnosing of psychotic disorder}

A E Gangat

Psychiatry Department, Nelson Mandela School of Medicine, University of KwaZulu-Natal, Durban, South Africa profgangat@gmail.com

The objective of the article is to throw light on a clear meaning of the terms psychosis and psychotic symptoms/ features. The diagnostic criteria in relation to a number of symptoms and duration are also studied. A careful study of the Diagnostic and Statistical Manual of Mental Disorders, 5th edition (DSM-5) (American Psychiatric Association) and the International Classification of Diseases (World Health Organization) indicates that there is no clear definition of the terms psychosis and psychotic symptoms/psychotic features. Kaplan and Saddok's psychiatry text book, a widely used reference book, also is confusing and imprecise. The American Psychiatric Association's definition of psychosis is even more elaborate and unclear. Issues pertaining to defining, classifying and diagnosing psychotic disorders, including schizophrenia, are discussed. These issues, including duration and number of symptoms required to make a diagnosis, are explored. The presentation is expected to generate strong discussion and debate.

The side-effects of sick leave: Are psychiatrists unintentionally harming their patients?

$\underline{\text { C Grobler, }}{ }^{*} \mathrm{~N}$ Smith

Walter Sisulu University, Mthatha, Eastern Cape, South Africa

*dr.stof@mweb.co.za

The Global Burden of Disease study ranks the magnitude of different health problems using a single measure, the disability-adjusted life year (DALY). This combines the years of life lost (YLL) from premature death and years of life lived with disabilities (YLDs). Unipolar depressive disorder is the third leading cause of disease burden, accounting for $4.3 \%$ of the global burden of disease. The estimates for low- and middle-income countries are $3.2 \%$ and $5.1 \%$, respectively. Current predictions indicate that by 2030 depression will be the leading cause of disease burden globally. When only the disability component is taken into consideration in the calculation of the burden of disease, mental disorders account for $25.3 \%$ and $33.5 \%$ of all years lived with a disability in low- and middle-income countries, respectively. The cost of sick leave runs into billions of rands every year in South Africa (SA).
According to the August 2013 Adcorp Index; 3.96 million workers were absent because of sickness during the year, compared with 700000 in 2000, an increase of 466\%. Sickness certification has been put under the spotlight in Europe, particularly Norway, Sweden, and the UK, where regulations for the issuing of sick leave are being reviewed in order to reverse the 'sick note culture'. People generally have the notion that, in order to sufficiently recover from ill health, they need to refrain from work. This is not always the case and recovery is often faster and more successful if people continue doing some work while recovering. The longer people are unemployed, the harder it is for them to find employment and successfully reintegrate into the working environment. Those who are unemployed for longer periods of time are at greater risk of mental and physical illness.

Conclusion. Issuing sick certificates has an effect on a country's economy, and thoughtless re-issuing of sick certificates without consideration of medical reasons as to why inactivity is necessary, may turn out to be detrimental to a patient's wellbeing. When completing a medical certificate, discuss the benefits as well as the potential detrimental effects of sick leave with patients. Obstacles with regard to returning to work should be pre-empted and addressed early on in the course of the mental illness. Taking into consideration current perspectives on the issuing of sickness certification, the clear evidence of the beneficial effects of working and changing global trends among practitioners, the time has come for SA doctors to re-evaluate their practices regarding the issuing of sick certificates. The message is clear: In the majority of cases, working is good for people, and work absence is not.

\section{Reflections on couples and couple work \\ O F I Habib}

Psychiatrist in private practice

habib@telkomsa.net

'Love and work ... work and love; that is all there is.' - Sigmund Freud This is an attempt to weave together three related narratives: (i) a psychiatrist revealing some aspects of his personal and professional identity pertaining to his style of work; (ii) sharing his philosophy, views, understanding and experiences regarding the nature of marriage, intimate relationships and relationship discord as derived from clinical practice; and (iii) concretely describing and demonstrating his clinical approach to couples in trouble. Through the study of clinical cases, it will be argued that a significant number of patients who present with depressive and anxiety disorders, are really masking serious interpersonal difficulties. This workshop-like presentation will further attempt to do the following: (i) provide a practical schema for marital (or relationship) history taking; (ii) provide suggestions as to how to approach partners to engage in couple work; (iii) discuss and demonstrate concrete framework on how to engage with troubled relationships - rules, boundaries, conflict management, communication, issues of reconciliation, separation or divorce, etc.; (iv) through the use of enactments and skits, the nature, challenges and usefulness of this kind of approach will be demonstrated.

\section{Cannabis in South Africa: Back to basics}

V Hitzeroth, ${ }^{*}$ L Kramer 
Stellenbosch University, Cape Town, and private practice in Bellville, South Africa

*psych1solway@eccsystems.co.za

Background. The ongoing debate regarding cannabis legalisation in South Africa (SA) is continuing relentlessly. Nationally, SA has had the recent publication of the South African National Cannabis Working Group findings in 2013, as well as the introduction to parliament of the Medical Innovation Bill which seeks to legalise the growing, production, distributing, using, prescribing and advertising of cannabinoids for medical treatment. On the international front, cannabis policy is continuing to be reviewed and adapted in various countries with subsequent changes in relevant legislation. It is thus clear that SA clinicians require up-to-date information, clinically relevant knowledge, as well as the theoretical and practical skills to treat our cannabisusing clients effectively, correctly guide their families and continue to contribute to the national debate regarding cannabis in an informed and coherent manner. As psychiatrists, we have to be aware of current thinking with regard to the risks and benefits, policy options and their specific implications for our patients, our profession and our country.

Method. A review of the worldwide literature with specific relevance to the most recent publications on cannabis, its clinical risks and benefits and other relevant themes in the international debate regarding the legalisation of cannabis.

Results. Findings of this review will address the following questions:

(i) basic cannabinoid physiology and psychopharmacology

(ii) cannabis potency; (iii) cannabis, adolescence and neurodevelopmental implications; (iv) cannabis and cognition; $(v)$ cannabis and mental health; (vi) cannabis and physical health; (vii) legalising, decriminalising and other jargon; (viii) international examples of various policy approaches (USA, Netherlands, Portugal, Spain, etc.); (ix) comparison with various other drugs of abuse (specifically nicotine and alcohol); and $(x)$ implications for SA.

Conclusion. Listeners to this presentation will have access to an evidence-based review of cannabis, so as to contribute to the national debate in an informed and meaningful manner.

\section{Dementia, advance directives and associated ethical challenges}

V Hitzeroth

Stellenbosch University, Cape Town, and private practice, Bellville, South Africa

psych1solway@eccsystems.co.za

Background. Alzheimer's dementia is becoming increasingly common as our modern population ages. The dementing process is characterised by an insidious onset and a decline in numerous cognitive domains. This results in progressive loss of functioning and ultimate incapacity and complete disability. In order to direct their future medical care, many individuals turn to an advance directive in the hope of providing guidance to their healthcare professionals when they are incapacitated and cannot do so. While advance directives carry no legal standing, they are relied upon to assist in medical decision-making. Yet, their ethical standing remains unclear and continues to be debated and challenged.

Method. In order to examine the ethical arguments relevant to the moral standing of advance directives a focused literature review on relevant themes was conducted.

Results. This presentation will offer the listener a number of arguments that support the moral standing of advance directives, namely autonomybased and beneficence-based arguments. Furthermore, this presentation will present a number of arguments that challenge the moral standing of advance directives, namely the informed-consent argument, loss-ofpersonality argument and the false-inference argument.

Conclusion. The debate surrounding the moral standing of advance directives continues unabated. The ethical arguments referred to in this debate remain persuasive. Every practising clinician should familiarise themselves with the relevant ethical arguments in order to provide the best care for their patients and to be able to assist the family in this challenging end-of-life situation.

(This presentation is based on an assignment for Module 3 of the MA (Medical Ethics and Law) at the University of Keele, UK.)

\section{Prescribing hypnosedatives - getting back to the basics M Huthwaite}

University of Otago, Dunedin, New Zealand

mark.huthwaite@otago.ac.nz

Background. Sleep disturbance is common in people with a psychiatric illness. Insomnia is a cardinal diagnostic criterion for major depressive episode, manic episode, and dysthymic, post-traumatic stress and generalised anxiety disorders. The relationship between disturbed sleep and the major psychiatric disorders may be bidirectional with a causal path leading from abnormal sleep to psychiatric disorders and vice versa. The psychopharmacological management of disturbed sleep in psychiatric illness can lead to complex prescribing regimens, off-label prescribing, altered sleep architecture and even drug dependence.

Objective. To identify the prescribing patterns in both inpatient and community samples and link our findings to the pharmacodynamic underpinnings of these prescribing choices.

Method. This project gathered data on hypnosedative prescribing patterns in outpatient psychiatric patients by examining all the clinical case records from two community mental health services in Wellington, New Zealand. Mental health service users (consumers) were also interviewed using a semistructured interview to explore their knowledge of the hypnosedatives they were being prescribed and their preferences in this regard.

Results. The data identify patterns of hypnosedative prescribing. Of the 318 participants over one-third (35.2\%) were prescribed at least one hypnosedative, of whom two-thirds (69\%) received this medication for more than 3 months. Another statistically significant finding was that more women $(45.3 \%)$ than men $(27.4 \%)$ and more patients with non-psychotic than psychotic disorders received hypnosedatives. Twothirds of those interviewed reported that they found the hypnosedative medications to be beneficial with $80 \%$ reporting that they fell asleep in less time, $58 \%$ woke less often, $61 \%$ noted that they slept longer and $59 \%$ felt more rested on awakening. However, nearly half reported some grogginess on waking and about a quarter reported being less active during the day as result. Linking the most commonly prescribed hypnosedatives to their pharmacodynamic and pharmacokinetic properties throws some light onto the rationale behind these research findings. 
Conclusion. Hypnosedative prescribing in psychiatric illness is common and often so prolonged as to breach guidelines. The usual community pattern of women being prescribed more frequently was replicated in this sample of psychiatric outpatients, but a contradictory finding of younger patients receiving more hypnosedatives emerged. The rationale for prescribing should be based on a sound knowledge of the pharmacodynamics and pharmacokinetics of the hypnosedatives. In the face of concerns about the use of hypnosedatives, it behoves practitioners and service users to consider alternatives, particularly in light of our research finding that more than half of mental health consumers wished to stop using hypnosedatives.

\section{The attitudes of doctors in South African teaching hospitals towards mental illness and psychiatry}

$\underline{\text { K L Jury }},{ }^{1 *}$ G Del Fabbro ${ }^{2}$

${ }^{1}$ University of the Witwatersrand, Johannesburg, South Africa

${ }^{2}$ Forensic psychologist in private practice

*kerryleighbalson@gmail.com

Background. Mental illnesses are highly prevalent worldwide. The majority of mentally ill individuals are reintegrated into society, where they often encounter stigma and discrimination. Stigmatisation by the community is well-known and highly researched. However, fewer studies have investigated the attitudes of medical doctors towards mental illness and no such study has been done in South Africa (SA). On reviewing the literature it is evident that negative attitudes also exist towards psychiatry as a profession; however there are limited data available on this topic.

Objective. To determine the attitudes of psychiatric and nonpsychiatric doctors towards people with mental illness and towards psychiatry as a profession.

Method. This was a prospective study in the form of a self-administered questionnaire, which was distributed to medical doctors at five teaching hospitals in Gauteng, SA. All qualified doctors working at the selected institutions were eligible to participate in the study, regardless of their level of experience or specialist field. The questionnaire investigated their attitudes towards three mental illnesses: depression, schizophrenia and borderline personality disorder, and towards psychiatry as a profession. A convenience sampling method was used and two different questionnaires were distributed, one to the psychiatric and the other to the non-psychiatric doctor group.

Results. A total of 531 doctors (16.4\% psychiatric and $83.6 \%$ nonpsychiatric) completed the questionnaire. Overall, the doctors' attitudes were more negative towards persons with schizophrenia and borderline personality disorder than towards those with depression. For all three of the mental illnesses in question more than $50 \%$ of the doctors felt that persons would improve with treatment; however, less than one-third felt that they would ever recover fully. The non-psychiatric doctors' attitudes were more negative towards persons with schizophrenia and depression than the psychiatric doctors, with significantly more non-psychiatrists agreeing that such persons are unpredictable, dangerous and hard to talk to. More than $70 \%$ of the psychiatric group felt that non-psychiatric doctors considered psychiatrists to know less than other doctors and psychiatry to be an unimportant specialty. However, less than $11 \%$ of the non-psychiatric doctors actually held these negative views.
Conclusion. Overall, doctors did hold negative attitudes towards mental illness. However, the psychiatrists were noted in a number of instances to be significantly more positive in their attitudes towards mental illness than their non-psychiatric colleagues. In addition, a large proportion of the psychiatrists had encountered stigma towards their profession and it is clear that they have misconceptions about other doctors' attitudes towards them and their profession.

\section{Borderline personality disorder in adolescents}

\section{E Karim}

Division of Child and Adolescent Psychiatry, Nelson R Mandela School of Medicine, University of KwaZulu-Natal, Durban, South Africa

karime1@ukzn.ac.za

High rates of suicide and non-suicidal self-injury (NSSI) in adolescents are viewed as major public health concerns. International research has demonstrated strong associations between these behaviours and borderline personality disorder (BPD). A recent local study, by the author, reviewed patients under 18 years of age, who had been hospitalised as a result of a suicide attempt or NSSI. The study revealed that while the presence of borderline traits was often noted in this group of patients, a formal diagnosis of BPD was infrequently made. This finding was consistent with research pointing to a reluctance on the part of clinicians to diagnose BPD in children and adolescents. Many reasons have been proposed to account for this finding, including stigma, beliefs that personality traits are still developing and should not or cannot be diagnosed in those under the age of 18 , perceived difficulties in diagnosing symptoms in this age group and beliefs that some BPD criteria may overlap with normative developmental processes. It is against this background that the following questions are considered about BPD in adolescents: (i) do our diagnostic systems permit a diagnosis of BPD in adolescents?; (ii) what is the prevalence of the diagnosis?; (iii) what is the significance of the diagnosis?; (iv) what are the symptoms in this age group, and are there gender differences?; $(v)$ is the diagnosis reliable, valid and stable?; (vi) what are the implications for treatment? This presentation seeks to address these questions by reference to the literature.

Conclusion. There may be a justifiable reluctance on the part of some clinicians to label adolescents with a potentially highly stigmatising disorder. Evidence suggests that BPD among adolescents is common in clinical settings, shows commensurate validity and reliability to adult BPD and has far-reaching implications for the patient. A failure to consider personality disorder in adolescents could lead to underdiagnosis, under-treatment or potentially inappropriate treatments.

Experience of receipt of care by patients with psychosis receiving treatment at complementary and alternative practitioners facilities: Report from the PAMD project

$\underline{\mathrm{L} \mathrm{Kola}_{2}}{ }^{*}$ V Makanjuola, O Gureje

Department of Psychiatry, University of Ibadan and University College Hospital, Ibadan, Nigeria

*lola_kola2004@yahoo.com

Background. Studies have reported that as many as $70 \%$ of patients receiving treatment in orthodox mental health facilities in Africa have earlier consulted and received treatment from complementary 
and alternative health practitioners (CAP). Complementary and alternative practices have been associated with numerous harmful practices including chaining and beating of patients among others. Few reports have considered the perception of the patients of their experience of receipt of care at CAP facilities. In a study conducted in three African countries, we explore the perception of the experience of receipt of care by patients receiving treatment from CAP facilities. Method. Qualitative study design using in-depth interview of patients with psychosis who have recovered sufficiently to be interviewed from CAP facilities randomly selected from the list obtained from an earlier mapping exercise. The interview guide was adapted from the Mini International Neurohsychiatric Interview and has questions on the experience of receipt of care, as well as satisfaction or otherwise with care received.

Results. Ninety-seven in-depth interviews were conducted with male and female patients. All the patients expressed satisfaction with the treatment received. Among the respondents who had experienced physical restraint, the majority felt it was justified. However, only two of the respondents said they would recommend similar treatment to others. Conclusion. While appreciating the level of care received from the CAP facilities, patients detest the harmful practices experienced at these facilities.

Development, adaptation and validation of the inter-UN OneHealth tool for estimating financial costs and health impacts of scaling up mental health services in South Africa $\underline{\mathrm{C} \text { Lund, }}{ }^{1 \star}$ S Docrat, ${ }^{1} \mathrm{D}$ Chisholm, ${ }^{2} \mathrm{M}$ Heslin, ${ }^{3}$ I Peterson ${ }^{4}$

${ }^{1}$ Alan J Flisher Centre for Public Mental Health, Department of Psychiatry and Mental Health, University of Cape Town, South Africa ${ }^{2}$ Department of Mental Health and Substance Abuse, World Health Organization, Geneva

${ }^{3}$ Department of Health Services and Population Research, Institute of Psychiatry, King's College, London, UK

${ }^{4}$ School of Applied Human Sciences, University of KwaZulu-Natal, Durban, South Africa

*crick.lund@uct.ac.za

Background. Resources for mental health care in low- and middleincome countries (LMICs) are in short supply, unevenly distributed and inefficiently used. Most government funds are directed towards the running costs of mental hospitals, which curb the development of more equitable and cost-effective community-based services.

Objective. The objective of the EMERging mentAL health systems in low- and miDdle-income countries (EMERALD) project is to improve mental health outcomes in LMICs by developing a clearer understanding of how to strengthen mental health systems. To address the question of resource adequacy, an integrated health systems resource planning tool for mental, neurological and substance use (MNS) disorders is being developed. This work builds on the OneHealth Tool (OHT), developed by international costing experts from the World Health Organization (WHO) and other United Nations (UN) agencies to strengthen health system analysis, costing and financing scenarios at country level. To our knowledge, MNS disorders and programmes have not been integrated into the OHT, and calculations of the resource needs for South Africa (SA) have not been conducted using this model.
Method. Vertical disease-specific service needs and intervention strategies for selected MNS disorders are mapped and integrated with horizontal health system components (e.g. human resources, information systems and infrastructure). Input data are drawn from a variety of SA sources, including national mental health plans, epidemiological surveys and mental health services research, supplemented by semi-structured interviews with programme managers in the SA Department of Health.

Results. The study identifies and quantifies human, infrastructural, informational and financial resource inputs, and estimates the costs of scaling up a core package of mental health services for depression, psychosis, alcohol use disorders and epilepsy, and the likely health impacts until 2035 in SA.

Conclusion. The OHT provides a means of transparently calculating the costs and likely health impacts of scaling up mental health services in South Africa, based on the best available data. The findings of this study can facilitate integration of mental health programme-specific plans into broader national health plans. Key gaps in our current data and the limitations of the assumptions in the model are discussed.

\section{Food insecurity and depression in refugees in Durban}

V Maharaj, ${ }_{2}^{*}$ M Mhlongo, L Thela, A Tomita, C Labys, J Burns

Department of Psychiatry, University of KwaZulu-Natal, Durban, South Africa

*allopi@webmail.co.za

Background. Since South Africa (SA)'s transition to democracy in 1994, we have become an increasingly popular destination for refugees; however, as a result of current poverty and political instability, refugees have become vulnerable to a host of psychosocial stressors. There is a paucity of data on common mental disorders (depression, anxiety and post-traumatic stress disorder), and risk factors for the development of these disorders in refugees in low- and middle-income countries.

Objective. To generate epidemiological data, as well as investigate the risk factors associated with the development of anxiety and depression in refugees in Durban, SA.

Method. A prospective study using a cross-sectional design was conducted. Three hundred and thirty-five (335) consecutive nonSA adults attending the Dennis Hurley Centre for refugees were interviewed during July 2103 and April 2014 using a sociodemographic questionnaire, the Hopkins Symptom Checklist (HSC), and the Food Security Scale. The association between food insecurity and anxiety and depression was investigated using logistic regression.

Results. The prevalence rates of anxiety and depression (using cut-offs of $\geq 16$ on the HSC anxiety subscale, and $\geq 24$ on the HSC depression subscale) were $50 \%$ and $54 \%$, respectively. After controlling for other sociodemographic risk factors in a logistic regression analysis, food insecurity over the last 12 months was significantly associated with a higher risk of both anxiety and depression. Individuals with food insecurity were $\sim 3$ times more likely to be anxious and five times more likely to be depressed than their food-secure counterparts.

Conclusion. This study reports important new data on the links between food insecurity as a proxy for poverty and common mental disorders in a previously unresearched but vulnerable population (refugees) living in SA. 
HIV risk behaviour in serious mental illness with and without co-occurring substance use disorders: Findings in a South African sample of inpatients

A Moodley, ${ }^{1 *} \mathrm{H}$ Temmingh, ${ }^{2}$ J Joska, ${ }^{2} \mathrm{D}$ J Stein ${ }^{2}$

${ }^{1}$ Department of Psychiatry, Stellenbosch University, Cape Town, South Africa

${ }^{2}$ Department of Psychiatry and Mental Health, University of Cape Town, South Africa

*moodley.anesh@gmail.com

Background. People with serious mental illness (SMI) and co-occurring substance use disorders (SUD), or dual diagnosis, are at increased risk of HIV exposure because of a higher likelihood of engaging in sexual and other risk behaviours. Little is known about the association between dual diagnosis and HIV-related sexual risk behaviour, in particular the role of methamphetamine use. We examined the association between HIV risk behaviour and SUDs including methamphetamine use in a sample of patients with serious mental illness admitted to an inpatient psychiatric unit.

Method. We determined substance use severity using the Clinician Alcohol Use and Drug Use Scales. Sexual risk behaviour was determined by a multi-item risk questionnaire. The primary outcome was the rate of sexual risk behaviours endorsed on the risk behaviour questionnaire for the 6-month period prior to hospital admission. We analysed data using multivariate negative binomial regression modelling.

Results. In the total sample of 96 participants, $59.4 \%$ had a diagnosis of a schizophrenia spectrum disorder, followed by bipolar disorder (25\%) and substance-induced mood and psychotic disorders (15.6\%). A total of $63.5 \%$ of participants had at least one SUD, with a methamphetamine use disorder occurring in $59 \%$ of participants with a SUD. As many as $67.7 \%$ of participants endorsed at least one item on the HIV-risk questionnaire. We found a significantly higher rate of risk behaviours in participants with any SUD (rate ratio $=1.77,95 \%$ confidence interval (CI) $1.19-2.16$ ) and in those who had a methamphetamine use disorder (rate ratio $=1.50$, $95 \%$ CI 1.05 - 2.14). After adjustment for demographic and associated clinical variables this association remained significant for participants with any SUD (rate ratio $=1.79,95 \%$ CI $1.26-2.53$ ), but was no longer significant for the group with methamphetamine use disorders (rate ratio $=1.09,95 \% \mathrm{CI} 0.75$ - 1.58). The rate of sexual risk behaviours remained significantly higher in the adjusted models for participants who used alcohol and other illicit drugs other than methamphetamine (rate ratio = 1.75 , 95\% CI 1.22 - 2.52).

Conclusion. Our findings correspond to previous research on HIV risk behaviour and its association with substance use disorders. The role of alcohol and other drug use disorders in the pathway between methamphetamine use and sexual risk behaviours warrants further research. Screening for sexual risk behaviour and offering appropriate HIV counselling may assist harm reduction in patients with SMI and co-occurring SUDs.

Factors associated with recidivism: A retrospective analysis of state patients admitted to Sterkfontein Hospital N Morgan, ${ }^{\star}$ G Del Fabbro

University of the Witwatersrand, Johannesburg, South Africa

*nirvana.morgan0@gmail.com
Background. This study examined common factors associated with recidivism among patients at Sterkfontein Hospital. Demographic, clinical and criminological factors of a recidivist group were compared with a non-recidivist group with the intention of understanding to what extent these factors might determine the risk of re-offending.

Method. A retrospective case file review of 293 inpatients and a random selection of 120 outpatients were conducted. For the purpose of the study a patient was classified as a recidivist if an additional charge or act of violence was added to the file while the patient was on leave of absence. Of the inpatients only those who met the criteria for recidivism were included in the study. All 120 randomly selected outpatients were included. Demographic, clinical and criminological data were captured for all patients.

Results. Of the 293 inpatients 60 patients met the criteria for recidivism. Twenty out of 120 outpatients were classified as recidivists. Thus 80 recidivists were compared with 100 non-recidivists. Using the $\chi^{2}$ and Fischer's exact test substance use disorder, antisocial personality disorder, an index offence of assault and inward adverse events were found to be predictors of recidivism $(p<0.05)$. Using logistic regression analysis the odds of a recidivism in a patient with an index offence of assault was 8.4 (95\% confidence interval (CI) 1.6 - 43.1) times that of one who did not commit assault as an index offence. The odds of recidivism for patients with cannabis use was 2.8 (95\% CI $1.3-6.0)$ and for patients with inward sexual offence was 17.2 (95\% CI 2.0 - 150).

Conclusion. This study suggests that state patients with comorbid substance use disorder and antisocial personality disorder are at higher risk for reoffending. Clinicians should also be aware of the potential risk among state patients with a charge of assault and patients who are found to be involved in inward adverse events. Important criminal history factors and certain clinical factors, however, could not be interpreted because of large amounts of missing data in patients' files.

\section{Celebrated journalist and author shares her experience of depression}

M Motlana, ${ }^{*}$ L Mountany, Z Mahomedy

Psychiatry M Powered

*drmashadi@gmail.com

Interview with author Redi Thlabi - book review: Endings and Beginnings: At the conclusion of the session, the participant should: (i) be able to understand the 'lived experience of mental illness'; (ii) have increased understanding of the role celebrities can play in raising awareness of mental illness; (iii) be encouraged to think of the causal factors of mental illness against the backdrop of our diverse socio-political history. The public sharing of a celebrity's experiences with mental health issues has been shown to be an effective tool for raising awareness. In this workshop we will interview Redi Direko about her book and explore the theme of her depression and how psychosocial factors contributed. We will seek to understand how a celebrity sharing these experiences helps in some way to increase the acceptability of mental illness in our society. We explore Redi's traumatic loss of her father and her search to understand a pivotal character in her life, in the shadowy figure of Mabegzo, a gangster in the community. Rethink.org, an NGO in the UK, commissioned a 


\section{ABSTRACTS}

survey on 2000 adults to test what myths were rife in the community and found that while celebrities such as Catherine Zeta-Jones and Stephen Fry helped to raise awareness on bipolar disorder, the stigma surrounding the disorder persisted with less than $18 \%$ of respondents being prepared to share their diagnosis with their employer. In the workshop we will grapple with these findings, explore how we can deepen the understanding of mental illness in our communities and deal more effectively with the related stigma.

Correlation of GHQ-12 values with quality of life (QOL) of subjects with essential hypertension attending the outpatient clinic of the University of Port Harcourt Teaching Hospital (UPTH)

A K Nkporbu, ${ }^{*}$ P C Stanley

Department of Neuropsychiatry, University of Port Harcourt Teaching Hospital, Port Harcourt, Nigeria

*nakpigi2008@yahoo.com

Background. Essential hypertension, a non-communicable disease, is fast assuming an epidemic dimension. Owing to the chronic nature of this condition, it commonly associated with psychiatric symptomatology and its attendant comorbidity.

Objective. The objective of this study, therefore, was to determine the relationship between the 12-item General Health Questionnaire (GHQ-12) scores and quality of life (QOL) in subjects with essential hypertension.

Method. Following ethical approval from the appropriate committee of the hospital and informed consent from the participants, 360 subjects making up the study group (hypertension) were recruited based on the study's inclusion and exclusion criteria. A pilot study had been conducted. Subjects were given the sociodemographic questionnaire, GHQ-12, and the brief version of the WHO Quality of Life instrument (WHOQOL-Bref) which were all self-administered. The data were analysed using the SPSS version 16 statistical package. Confidence interval (CI) was set at $95 \%$ while a $p$-value of less than 0.05 was considered statistically significant.

Results. From the study, the mean (standard deviation) QOL domain scores for the hypertensive group were as follows: 50.97 (14.67), 55.20 (22.19), 54.51 (22.13), 50.01 (16.91) and 49.34 (22.44) for physical, psychological, social relationship, environment domains and general health facets respectively. Furthermore, GHQ-12 values were significantly inversely correlated with QOL. The only significant relationship with sociodemographic variables among the hypertensives was that physical health domain of QOL was significantly lower among the divorced and the widowed compared with other marital groups $(p<0.001)$.

Conclusion. The findings in this study indicate that essential hypertension is a chronic debilitating and disabling illness, associated with psychiatric symptomatology with higher psychic distress, as shown by GHQ-12 scores. It appears that hypertensives with higher GHQ-12 values were associated with significantly lower QOL in all domains than those with lower values. The results indicate that the management of hypertension should include attention to their mental health status and subjective QOL of these patients in order to enhance the quality of care.
Sociodemographic and clinical determinants of psychiatric comorbidity among people living with HIV attending the University of Port Harcourt Teaching Hospital

A K Nkporbu ${ }_{2}^{1 *}$ P C Stanley, ${ }^{1} \mathrm{~J} \mathrm{Phaeri}^{2}$

${ }^{1}$ Department of Neuropsychiatry, University of Port Harcourt Teaching Hospital, Port Harcourt, Nigeria

${ }^{2}$ Federal Neuropsychiatric Hospital, Enugu, Nigeria

*nakpigi2008@yahoo.com

Background. HIV, a communicable disease, is assuming an alarming epidemic dimension with a high level of psychiatric comorbidity and mortality. A number of sociodemographic and clinical determinants may be important in the psychiatric comorbidity outcome of this dreaded condition.

Objective. To determine the sociodemographic and clinical factors that may influence the level of psychiatric comorbidity associated with people living with HIV (PLWHIV) attending the retroviral outpatient clinic, University of Port Harcourt Teaching Hospital (UPTH).

Method. Following ethical approval from the appropriate committee of the hospital and informed consent from the participants, 230 subjects making up the study group were recruited based on the study's inclusion and exclusion criteria. A pilot study had been carried out. Subjects were given the sociodemographic 12-item General Health Questionnaire (GHQ-12), and WHO Composite International Diagnostic Interview (WHO CIDI).The sociodemographic/clinical questionnaire and GHQ12 were self-administered while the WHO CIDI was based on an interview by the researcher. The data were analysed using the SPSS version 16 statistical package. Confidence interval (CI) was set at $95 \%$ while a $p$-value of $<0.05$ was considered statistically significant.

Results. The study found a prevalence of psychiatric comorbidity of $17.3 \%$ among PLWHIV. Depressive illness had the highest prevalence of 47 (29.4\%). Among the control group, there was no significant relationship between the presence of psychiatric comorbidity and age group $(p=0.350)$, gender $(p=0.22)$, marital status $(p=0.07)$, level of education ( $p=043)$, income class $(p=0.81)$ and occupation. Persons who reacted with either 'very sad' or a 'wish to die' when they were informed of the diagnosis of the medical condition were significantly more likely to have psychiatry comorbidity $(p=0.001)$. There was also no significant relationship between age of onset of illness $(p=0.60)$, duration of illness $(p=0.73)$ and duration of treatment $(p=0.82)$. Persons who have suffered self-stigma were also significantly more likely to have psychiatric comorbidity ( $p=0.001$ ).

Conclusion. The findings of this study support the impression that HIV infection is a chronic debilitating illness, associated with psychiatric comorbidity, that is largely significantly influenced by a number of sociodemographic and clinical factors. The results support the call that the management of patients with HIV should include attention not only to their mental health status, but also the psychosocial and other clinical factors they may present with in order to enhance the quality of care.

Traditional healers in the treatment of mental illness: A systematic review

G Nortje, $^{1 *}$ B Oladeji, ${ }^{2}$ O Gureje, ${ }^{2}$ S Seedat ${ }^{1}$

${ }^{1}$ Department of Psychiatry, Stellenbosch University, Cape Town, South Africa 
${ }^{2}$ Department of Psychiatry, College of Medicine, University of Ibadan, Ibadan, Nigeria

*g.nortje@gmail.com

Background. Traditional healers form a major part of the mental health workforce in South Africa and worldwide. Despite this, there has been little systematic examination of their efficacy in treating mental illness. In this systematic review we focus on the efficacy of traditional healing, defined as healing modalities which explicitly appeal to supernatural or meta-physical explanations for illness or misfortune and which use magico-religious methods and rituals for treatment.

Method. Four medical and social science databases (Medline, Scopus, PsycArticles, Social Science Citation Index) were systematically searched by two reviewers, followed by reference chaining of potentially eligible articles. Key authors were contacted to suggest additional sources. Eligible articles were classified according to their level of evidence. Data describing outcome were extracted and summarised. Qualitative data describing treatment practices and potential therapeutic mechanisms were also extracted and grouped into themes.

Results. Traditional healers across the world use broadly similar models for illness, diagnosis and treatment. Many nonspecific aspects of treatment overlap with those used by allopathic psychiatrists. There are very few studies which rigorously evaluate the efficacy of traditional healers in treating mental illness. The evidence base consists largely of anthropological case series and anecdotes, with some exceptions. Traditional healers may relieve distress and improve well-being by providing meaning and context for illness, and by facilitating social engagement and improving coping strategies. Symbolism used in rituals and ceremonies facilitates a shift from the sick-role towards recovery. These techniques may be effective in disorders with prominent social and psychological components, but are less effective in psychotic disorders.

Conclusion. There is little good-quality evidence that traditional healers are efficacious in treating the symptoms of mental illness. However, the broader contributions of traditional healers to well-being and healing may be inadequately captured by conventional psychopathological rating scales. We outline the challenges of evaluating the efficacy of traditional healers in a systematic and rigorous way.

After integrating psychotropic medications into the care of people with mental disorders in a prayer camp in Ghana, why are all the chains not falling off?

A Ofori-Atta, ${ }^{1 *}$ F Baning, ${ }^{2} \mathrm{R}$ Rosenheck ${ }^{3}$

${ }^{1}$ University of Ghana Medical School, Ghana

${ }^{2}$ Pantang Hospital, Ghana Health Service, Ghana

${ }^{3}$ Yale University, USA

*angela.oforiatta@gmail.com

Background. Because the treatment gap for mental illness in Ghana is over $98 \%$ where conventional psychiatric treatment is concerned, we began to look closely at how care is accessed in Ghana. We learned that most Ghanaians visit herbalists, fetish priests, and faith healing camps, as well as state psychiatric hospitals for treatment. In many of these places there were reports of gross abuses of human rights. We therefore put together a clinical trial of psychotropic medication versus standard faith healing camp practices and ran the trial for a year. Our theory of change was that with the use of medication in the faith healing camp, patient health outcomes would improve, the chaining of patients would stop, and camp attendants' world views would change to a more biopsychosocial perspective from only a spiritual one.

Method. Residents of a sanatorium in a prayer camp in Ghana were screened for psychiatric disorders by a resident (medical officer) in psychiatry. Those diagnosed with mental illness (schizophrenia, mood or anxiety disorders) and requiring treatment were randomly assigned to receive either a daily dose of medication and regular prayer camp treatment (intervention group) or only regular prayer camp treatment (control group) for 7 weeks. Each patient was reviewed fortnightly. At the end of the period, all patients had the option to receive medication either at the prayer camp or, if discharged, through local health facilities. All were assessed by the Brief Symptom Inventory, Global Assessment of Functioning Scale, Lehman Quality of Life Interview and the Patient Health Questionnaire. Interviews were conducted with all staff members about their views of illness causation at weeks 1,9 and 18 weeks to assess change over time. Intervention and data collection are ongoing.

Preliminary results. Observations already indicate better patient outcomes. However, the focus of attention of this article is on why these improvements in patient health outcomes are not fully reflected in the de-chaining of patients in the camp.

Conclusion. Findings shall inform policy on integration of mental health care in indigenous community settings in Ghana.

\section{Positioning a new cadre of community workers in the mental health system of a low-resource country: The case of Ghana A Ofori-Atta, ${ }^{1 *}$ R Ketor, $^{1}$ E Bradley ${ }^{2}$ \\ ${ }^{1}$ University of Ghana Medical School, Accra, Ghana \\ ${ }^{2}$ Yale University, New Haven, USA \\ *angela.oforiatta@gmail.com}

An estimated 19\% of the population in Ghana suffers from moderate to severe psychological distress. In 2005, only 32875 people were hospitalised or given outpatient consultations in Ghana, representing approximately a $98 \%$ treatment gap. Mental health disorders also inflict a large cost on Ghana's economy, estimated at a 7\% loss in potential gross domestic product (GDP). A major problem with Ghana's mental health system is shortage of mental health professionals and an absence of support staff below community psychiatric nurses (CPNs) (of whom there are approximately 300). Each year, however, the University of Ghana graduates at least 300 students with a bachelor's degree in clinical, community, social, and health psychology who could be deployed as case finders, offer psychosocial support to patients, provide mental health education in communities and schools, execute public mental health preventive programs, and liaise between psychiatry wings of regional hospitals and patients' families, through the national service programme (a compulsory 1-year placement of all new graduates in the Ghanaian public service). This would immediately make a few hundred community workers available each year in mental health. The programme was launched in August 2012 and approximately 250 graduates were posted to work with CPNs around the country. In 2013, fewer graduates were posted because of changes in the national service secretariat which owns the national service. This article documents the experience of introducing this new cadre of staff, and assesses the impact of the psych corps on 


\section{ABSTRACTS}

community services using interviews with nurses and some members of the psych corps themselves. The overall consensus was that the effects were positive. More careful planning, implementation and deft political manoeuvring are required if the psychology profession is to accept task shifting as the way forward to build the profession and its services.

\section{Cognitive performance during the first year of treatment in} first-episode schizophrenia: A case-control study

$\underline{\text { R Olivier, }}{ }^{*}$ S Killian, B Chiliza, L Asmal, R Schoeman, P P Oosthuizen, M Kidd, R Emsley

Department of Psychiatry, Stellenbosch University, Cape Town,

South Africa

*olivier.r@vodamail.co.za

Background. Several questions remain unanswered regarding the magnitude and time course of cognitive improvement in response to antipsychotic treatment. The purpose of this study was to assess changes in cognitive performance in antipsychotic-naive or minimally medicated patients with first-episode schizophrenia during the first 12 months of treatment, in a case-control design. Patients were treated according to a fixed protocol with flupenthixol decanoate depot injection, according to a standard algorithm. The primary outcome measure was change in MATRICS Cognitive Consensus Battery (MCCB) composite score over 12 months.

Method. The sample comprised 92 patients and 100 healthy controls matched for age, sex, ethnicity and educational status. Cognitive function was assessed by means of the MCCB.

Results. A mixed-effects model identified a significant between-group difference over time $(p \leq 0.0001)$ for the MCCB composite score, with patients showing clear improvements from baseline to month 6 but not from month 6 to month 12, but remaining lower than control scores. For the other MCCB domains there were group differences at adjusted significance level for attention and vigilance $(p \leq 0.0001)$, visual learning ( $p \leq 0.0001)$, verbal learning ( $p=0.005)$, and working memory $(p \leq 0.0001)$, but not for reasoning and problem solving $(p=0.04)$, speed of processing $(p=0.03)$, and social cognition $(p=0.06)$. There were moderate correlations between change in MCCB composite score and change in symptomatology as assessed by positive and negative syndrome score (PANSS) factor-analysis derived domains.

Conclusion. Substantial improvements in cognitive function were observed over and above a practice effect, and were significantly correlated with improvements in psychopathology and functionality.

A cross-sectional study investigating the concurrent validity of the Montreal Cognitive Assessment (MoCa) tool with the MMSE in patients with schizophrenia

P Oosthuizen, ${ }^{*} \mathrm{H}$ Temmingh, I Lewis, H Thornton

University of Cape Town, South Africa

•flippieoosthuizen@yahoo.co.uk

Background/Objective. Cognitive impairment is a key clinical feature and prognostic factor in schizophrenia. The Folstein Mini Mental State Examination (MMSE) is the most widely used cognitive screening tool in South Africa (SA). However, the MMSE has several limitations as it was designed as a screening tool for Alzheimer's dementia. A suitable screening tool of cognitive functions in schizophrenia should include a more extensive exploration of executive function and memory. The Montreal Cognitive Assessment (MoCa) tool seems to be a possible alternative to the MMSE. The objective of this study was to explore the usefulness of the MoCa, when compared with the MMSE, in measuring cognitive impairment in patients with schizophrenia in an SA setting.

Method. We recruited patients with schizophrenia and controls who were friends or family of patients attending an outpatient psychiatry department. Inclusion criteria were age 18 - 55, at least 7 years of education, English-speaking, able to read and write. Both patients and controls underwent clinical assessment to confirm diagnosis (patients) and exclude any history of established neurological disorders, dementia, cognitive impairment or intellectual disability and psychotic symptoms (for controls). We measured psychotic symptom severity with the positive and negative syndrome score (PANSS) rating scale. Both the MoCa and the MMSE were administered on all patients. The primary outcomes were change on the MMSE and MoCa and the correlation between MMSE and MoCa. We constructed a series of multivariate linear regression models to adjust for confounders and to examine for potential effect modification.

Results. The total sample consisted of 40 participants with schizophrenia and 32 controls. Within the schizophrenia group we found significant negative correlations between the PANSS total and the MMSE (Spearman's $\rho=-0.55, p<0.001$ ) and the MoCa (Spearman's $\rho=-0.50, p<0.001$ ). In the adjusted models, schizophrenia patients had significantly lower sores on the MoCa $(\beta=-2.92, p=0.014)$, and on the MMSE $(\beta=-0.16, p=0.037)$. We found a significant positive correlation between the MMSE scores and the MoCa that persisted after adjustment for educational level $(\beta=1.26$, $p<0.001)$. However, participants with tertiary compared with primary education on average had significantly higher MoCa scores $(\beta=3.44$, $p=0.048)$. There was a significant positive correlation between MMSE and MoCa scores for both schizophrenia and control groups. We found no evidence of effect modification based on patient status in multivariate models (interaction effect: $\beta=0.27, p=0.303$ ).

Conclusion. The MMSE correlates well with MoCa. This appears to be independent of whether participants were patients or controls. However the correlation between the MoCa and MMSE appears to be influenced by educational level, with participants with tertiary education having significantly higher MoCa scores. Limitations include the lack of a gold standard cognitive instrument to establish criterion validity.

\section{Institutions, psychiatrists and society}

IS Parker

Department of Psychiatry and Mental Health, University of Cape Town, South Africa

john.parker@westerncape.gov.za

The early history of psychiatric institutions and their rapid expansion in the eighteenth to mid-twentieth century, as well as their role in the origins of the discipline of psychiatry, is examined in terms of its implications for modern psychiatry and the roles played by psychiatrists in society.

\author{
The rights of people living with mental illness to vote in \\ South Africa \\ IS Parker
}


Department of Psychiatry and Mental Health, University of Cape Town, South Africa

john.parker@westerncape.gov.za

Chapter 2, 8 (c) and (d) of the Electoral Act No. 73 of 1998 and its amendments in the Electoral Amendment Act 2013 state that persons declared by a high court to be of 'unsound mind' or declared 'mentally disordered' may not be registered on the voters' roll and also refers to those 'detained' under the Mental Health Care Act as excluded from the voters' roll. The effect of this law has been the almost total exclusion from the electoral process of a significant number of mental healthcare users, yet there has been little public interrogation of the issue. When they are more carefully considered, those aspects of the Electoral Act mentioned above are shown not to be consistent with the Mental Health Care Act, or with Curatorship Law, or with current understandings pertaining to the specificity of incapacity and the localisation of impairments. The question of 'capacity to vote' is examined against examples from the USA and European countries and in the context of the South African Constitution and of the United Nations Declaration on Human Rights.

\section{Clinical correlates and risk factors in first-episode psychosis in adolescents}

S Paruk, ${ }^{*}$ K Jhazbhay, K Singh, JK Burns

University of KwaZulu-Natal, Durban, South Africa

*myamod@telkomsa.net

Background. Approximately $40 \%$ of male patients and $23 \%$ of females have onset of schizophrenia before age 19, indicating a need for early identification of symptoms and risk factors in early-onset psychosis. Environmental factors such as early cannabis use and childhood abuse have been implicated as risk factors in biologically vulnerable adolescents for early-onset psychosis.

Objective. To examine the clinical characteristics and common risk factors associated with early-onset psychosis in an African adolescent first-episode psychosis (FEP) sample.

Method. Thirty-five adolescents aged 10 - 18 years with FEP were recruited from four psychiatric units in KwaZulu-Natal. Participants were assessed using a structured data sheet, positive and negative syndrome score (PANSS), Symptom Onset in Schizophrenia (SOS) inventory and the WHO Assist Questionnaire for substance use. Controls were recruited from the same sites and matched for age and gender.

Results. The demographic profile, age of onset, prodromal symptoms, nature of psychotic symptoms, diagnosis and substance use patterns are analysed in the FEP group. The presence of risk factors such as family history of mental illness, child abuse, and cannabis use are compared with a group of mentally ill adolescents without psychosis. Conclusion. Early-onset psychosis is associated with a poorer long-term outcome. Greater awareness of risk factors and clinical characteristics of adolescent-onset psychosis will assist in targeting modifiable risk factors in vulnerable adolescents such as substance use, promote early intervention and thus ultimately translate into improved outcomes.

Clinical and functional outcome of treatment-refractory first-episode schizophrenia

$\underline{\text { L Phahladira }}{ }^{*}$ R Emsley, L Asmal, B Chiliza
Department of Psychiatry, Stellenbosch University, Cape Town, South Africa

*lebogang.phahladira@gmail.com

Background. Most patients who experience a first episode of psychosis (FEP) respond to antipsychotic treatment, with up to $80 \%$ responding within the first year. Patients with ongoing psychotic symptoms are more likely to have a poor outcome and are less likely to benefit from rehabilitation efforts. Treatment-resistant schizophrenia is not a discrete entity; however, there are several predictors of poor outcome that have emerged from follow-up studies of schizophrenia patients. The objective of this exploratory study was to describe the trajectory and outcome of treatment refractoriness in FEP.

Method. In a prospective, longitudinal study of first-episode schizophrenia within the greater Cape Town area, 12 of the 130 subjects failed to adequately respond to standardised treatment with flupenthixol decanoate and were classified as treatmentrefractory. We conducted a cross-sectional descriptive assessment of these patients $(N=12)$ using the Positive and Negative Symptoms Scale for Schizophrenia, the Clinical Global Impression, Social and Occupational Functioning Assessment, the World Health Organization Quality of Life scale and the Neurological Evaluation Scale. The current treatment with antipsychotics and concomitant medications was recorded.

Results. In our sample, we identified demographic, clinical and functional factors associated with treatment refractoriness in schizophrenia. Clinical, functional and treatment outcome in these patients will be described. The limitations of the study are a small sample size and cross-sectional observation.

Conclusion. The evolution of treatment refractoriness in schizophrenia is poorly understood. While some patients are nonresponsive to antipsychotic treatment from the outset, others appear to respond initially, but after experiencing a relapse fail to improve to their previous levels.

Subjective memory complaints as a screening tool for cognitive impairment

$\underline{\text { S Ramlall, }}{ }^{1 *}$ J Chipps $^{2}$

${ }^{1}$ University of KwaZulu-Natal, Durban, South Africa

${ }^{2}$ University of the Western Cape, Bellville, South Africa

*ramlalls4@ukzn.ac.za

Background. There is increasing evidence that self-reported memory or cognitive problems are a potentially valid early clinical marker of brain and cognitive changes that may indicate cognitive impairment. With the anticipated increase in burden of dementia expected in developing countries, where dementia remains a low priority and resources are limited, the local utility and validity of subjective memory complaints (SMCs) is worthy of exploration.

Methods. In a three-stage cross-sectional study conducted in an old-age residential facility, 302 elderly aged 60 years and older were screened for dementia using subjective and objective measures of memory; 140 of the elderly who were screened were subsequently clinically assessed for a diagnosis of dementia or mild cognitive impairment (MCI). The $\mathrm{SMC}$ is a single domain screening measure assessing memory through 


\section{ABSTRACTS}

a 'yes/no' response to the question: 'Are you experiencing any difficulty with your memory?' Subjective Memory Complaint-Clinical (SMCC), compiled for the study, is distinguished from SMC by the specification of duration (1 year), frequency (at least once a week) and nature of the memory complaint in seven specified situations.

Results. Of the 320 participants, 140 (46\%) reported the presence of SMC. There was no significant association with race, $\chi^{2}=4.7, p=0.193$; gender, $\chi^{2}=0.8, p=0.438$; age, $\mathrm{U}=1.8, p=0.07$ or education, $\mathrm{U}=0.8$, $p=0.426$. The presence of at least one SMCC was reported by $80 / 140$ (57.1\%) participants with a significant association with race $(\mathrm{K}=7.9$, $p=0.048$ ). Both SMC and SMCC were significantly able to discriminate between dementia, MCI and control participants $(p=0.002$ and $p<0.001$ respectively). The SMCC also displayed higher sensitivity (90.9\% v. $63.6 \%)$, positive predictive value (PPV) (12.5\% v. 10.6\%) and negative predictive value (NPV) $(98.3 \%$ v. $94.6 \%)$ than the SMC. The SMCC had moderate discriminatory power to correctly classify those with and without dementia (area under the curve (AUC) $=0.717, p=0.004$ ) with a sensitivity of $90.0 \%$ and specificity of $45.7 \%$ as a screening measure for dementia. Two of the seven SMCC items were significantly able to discriminate dementia from non-dementia participants.

Conclusion. The performance of both the SMC and SMCC in our study was superior to that reported in the literature. The SMCC had moderate discriminatory power to correctly classify those with and without dementia with potential utility as a single-domain first-step screening test for cognitive impairment in the elderly.

\section{AAPAP special symposium}

$\underline{\text { S Rataemane }}$

Department of Psychiatry, University of Limpopo, MEDUNSA, South Africa

solomon.rataemane@ul.ac.za

The African Association of Psychiatrists and Allied Professions (AAPAP) supports the efforts made by SASOP in mental health information sharing, building bridges across African countries and providing support to less-resourced countries in various regions of Africa in mental health service development. As an example of cooperation, AAPAP has singled out contributions from five African countries outside of South Africa to demonstrate the nature of research done, the commonality of goals in service delivery, research and advocacy for mental health service delivery. The topics include care of patients with psychosis in Nigeria; anxiety and depression among orphaned adolescents in Kenya; factors associated with harmful drinking in Ethiopia; maternal mental health in Uganda; and factors influencing duration of untreated psychosis in Sudan.

Burnout and secondary traumatisation: Helping the helpers S Rataemane

Department of Psychiatry, University of Limpopo, MEDUNSA, South Africa

solomon.rataemane@ul.ac.za

Burnout is a state of emotional, mental, and physical exhaustion caused by excessive and prolonged stress. It occurs when you feel overwhelmed and unable to meet constant demands. As the stress continues, you begin to lose the interest or motivation that led you to take on a certain role in the first place. However, secondary traumatisation (also called vicarious traumatisation) is the stress resulting from helping or wanting to help a traumatised or suffering person. This is the cumulative transformative effect of working with survivors of traumatic life events. Vicarious traumatisation and burnout are on a continuum, with the latter being the end stage or ultimate failure to prevent or manage secondary traumatisation. This continuum will be discussed with reference to early identification and management of both elements in the context of psychotrauma, particularly in helping survivors of traumatic life events.

Prevalence and correlates of non-medical stimulant and related drug use in a sample of South African undergraduate medical students

M Retief, ${ }^{*}$ K Cloete, C Verster, J Harvey

Department of Psychiatry, Stellenbosch University, Cape Town,

South Africa

*retief.mari@gmail.com

Background. The non-medical use of prescription psychostimulants or cognitive-enhancing substances among healthy college students is a growing concern. This use appears to be particularly high among medical students. To our knowledge, no literature is available on the non-medical use of stimulants among South African (SA) medical students.

Objective. To determine the prevalence and correlates of nonmedical stimulant use, as well as subjective opinion on peer numbers using stimulants and university attitude towards stimulant use, among a sample of SA undergraduate medical students.

Method. A descriptive observational study was conducted by means of a self-report questionnaire. Second- and fourth-year medical students $(N=252)$ completed the questionnaire.

Results. Of the sample, 42 (17\%) reported a lifetime use of stimulants for non-medical purposes and 33 (85\%) of this group reported use within the past year. A total of six (2\%) students reported a diagnosis of attention deficit hyperactivity disorder (ADHD). In the group without a diagnosis of ADHD, non-medical stimulant use was associated with year of study $(p=0.03)$ and illicit substance use $(p=0.01)$. Most of the students in this group reported using stimulants to improve concentration.

Conclusion. Non-medical use of stimulants to improve concentration and academic performance is prevalent among the SA medical students sampled in this study. Further research at other institutions and under non-medical students would be helpful to assess the scope of this phenomenon.

Genetic variation and clinical variables contributing to schizophrenia in a founder population from South Africa

$\underline{\text { L Roos }}$

Department of Psychiatry, University of Pretoria, South Africa Erna.Fourie@up.ac.za

This talk will deal with findings of the genetic architecture of schizophrenia in an Afrikaner founder population and clinical related variables pertaining to this population. The initial research findings supported the appropriateness of the Afrikaner population for mapping complex traits using both linkage and linkage disequilibrium (LD) approaches. It was 


\section{ner
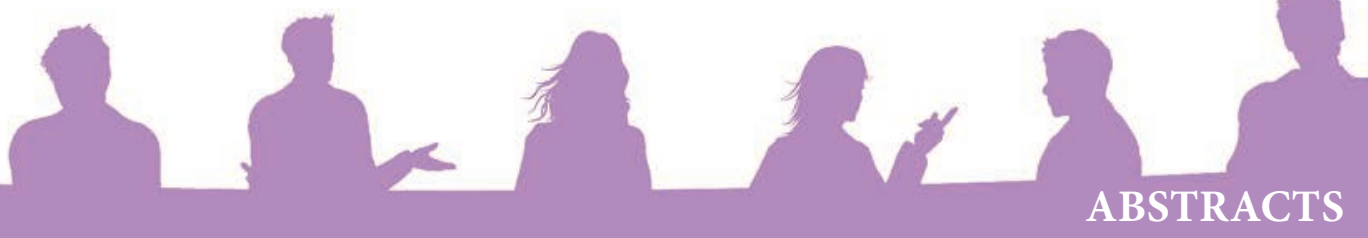

concluded that the results from our genetic study of schizophrenia in the Afrikaner sample will be applicable to other populations. Early nonpsychotic childhood deviance (in the first 10 years of life) distinguished a distinct subtype of schizophrenia patient and it may be a possible endophenotypic marker in schizophrenia but not in bipolar disorder. We addressed the role of the individual genes from the 22q11 locus (prototype CNV described in schizophrenia). Systematic screening of the 26 genes residing in this locus identified PRODH2, ZDHHC8, NOGO Receptor 1 (RTN4R) gene as contributing to schizophrenia risk associated with this region. Linkage genome-wide scans, using both less dense $(10 \mathrm{cM})$ and more dense scans $(2 \mathrm{cM})$, identified a locus on chromosome 1 and 13 . Recent fine mapping on chromosome 13q32-34 and brain expression analysis implicates MYO16 in schizophrenia. We confirmed the previous reported rate of $2 \%$ frequency of $22 \mathrm{q} 11$ deletions in adult schizophrenia Afrikaner patients and provide a two-stage screening protocol to identify these patients in clinical practice. Certain comorbid conditions such as marijuana use/abuse and obsessive compulsive disorders and symptoms as found in this population will be discussed. As the research progressed the emphasis changed from familial cases with the disease to sporadic cases (non-familial). We offered the first clear view of the genetic landscape of schizophrenia. We found that rare de novo structural mutations at many different loci are significantly enriched and contribute to schizophrenia vulnerability in sporadic cases with the disease. We also demonstrated that genes contribute to familial schizophrenia, while new mutations are less prominent. The study of schizophrenia in the Afrikaner founder population has helped to clarify the view of the genetic landscape of schizophrenia.

Neurocognition and imaging genetics in social anxiety disorder in the context of early developmental trauma

D Rosenstein, ${ }^{1 *} \mathrm{~S}$ Hemmings, ${ }^{1} \mathrm{~F}$ A Liato, ${ }^{1} \mathrm{~J}$ P Fouche, ${ }^{2} \mathrm{~J}$ Zwart, ${ }^{3}$ S Seedat ${ }^{1}$

${ }^{1}$ SARChI: PTSD, Department of Psychiatry, Stellenbosch University, Cape Town, South Africa

${ }^{2}$ Department of Human Biology, University of Cape Town, Department of Psychiatry and Mental Health, University of Cape Town and Department of Psychiatry, Stellenbosch University

${ }^{3}$ Department of Physics and Astronomy, University of the Western Cape, Bellville, South Africa

*rosensteind@gmail.com

A number of cognitive deficits have been found in social anxiety disorder (SAD). However, the impact of early-life trauma and genetics on brain morphometry and neurocognition in SAD has not been fully elucidated. A cognitive test battery was administered to determine cognitive performance in visual memory, verbal memory, attention and executive functioning in participants with SAD and early developmental trauma (EDT). The following genotypes were also investigated: 5-HTT, TPH-2, RGS-2, together with structural magnetic resonance imaging (sMRI) and regional brain network analyses. Individuals with SAD and EDT $(N=25)$, individuals with SAD without EDT $(N=24)$ and education-, handedness, gender- and age-matched healthy controls $(N=26)$ were compared. Bayesian and null hypothesis significance testing were used in the data analysis. Differences were found in verbal memory, executive functioning and motor performance between participants with SAD and EDT and healthy controls. Differences were also found in verbal memory in the SAD without EDT group compared with controls. No differences in cognitive performance were found between the SAD with EDT and SAD without EDT groups. sMRI differences were found mainly in the SAD with EDT group in the anterior cingulate, thalamus and caudate nucleus compared with the controls. There were also differences in the caudate nucleus between the SAD with EDT and SAD without EDT groups. Imaging genetics differences indicated a unique interplay between early trauma and genotypes. This study is unique in its comparative assessment of the effects of EDT on neurocognition and imaging genetics in individuals with SAD.

Low frequency of screening for metabolic syndrome in patients with severe mental illness at a general hospital psychiatric unit in Durban

$\underline{\text { S Saloojee, }},{ }^{1 *} \mathrm{~J}$ K Burns, ${ }^{1}$ A A Motala ${ }^{2}$

${ }^{1}$ Department of Psychiatry, Nelson $R$ Mandela School of Medicine, University of KwaZulu-Natal, Durban, South Africa

${ }^{2}$ Department of Diabetes and Endocrinology, Nelson R Mandela School of Medicine, University of KwaZulu-Natal, Durban, South Africa

*saloojees1@ukzn.ac.za

Background. Sub-Saharan Africa is experiencing the largest increase in the prevalence of type 2 diabetes mellitus and cardiovascular disease globally. Metabolic syndrome (MetS) is a cluster of risk factors for these conditions. There is a consistently higher prevalence of cardiometabolic disease among individuals with severe mental illness (SMI) compared with the general population worldwide. However, it is known from research in high-income countries that screening for MetS in patients with SMI is low. The objective of this study was to document the extent of the expected low frequency of testing for all the components MetS in patients with SMI in a low- to middle-income country.

Method. This was a cross-sectional study, undertaken from January to June 2012 on outpatients with SMI who were treated with antipsychotic medication for at least 6 months. The study measured the proportion of participants who were tested for MetS and the site of their general medical care in the previous year.

Results. The study included 331 (M:F;167:164) participants with a mean (standard deviation) age of 35.2 (11.98) years. The majority (78.8\%) were black South Africans. Only two subjects (0.6\%) were screened for all five components of MetS. Regarding the individual components, $99 \%, 0.6 \%, 3.9 \%$ and $1.8 \%$ were screened for raised blood pressure, abdominal obesity, hyperglycaemia, hypertriglyceridaemia and decreased high-density lipoprotein cholesterol, respectively. Fortytwo per cent relied on the psychiatric clinic for their general medical healthcare.

Conclusion. It is unacceptable that less than one per cent of our participants were screened for modifiable risk factors. These results therefore highlight the need for translating guidelines into action.

Depression among secondary school students in Ludewa district, Tanzania

M B Sebit, ${ }^{1 *}$ F Benedict $^{2}$

${ }^{1}$ Department of Psychiatry and Neurology, College of Medicine,

University of Juba, South Sudan 
${ }^{2}$ Department of Psychiatry, Njombe Town Hospital, Njombe, Tanzania *mbsebit@gmail.com

Background. Depression among secondary school children is common, and the lifetime prevalence of major depressive disorder in adolescents is estimated to be between $15 \%$ and $20 \%$, similar to the rates in the adult population, but it has never been rigorously addressed in Tanzania.

Objective. To determine the prevalence and sociodemographic factors related to depression among school children in Ludewa district, Tanzania.

Method. It was a cross-sectional community-based study design using a multistage cluster sampling technique and quantitative data collection methods. A total of 432 eligible secondary school students, 206 male, from Form One to Form Four classes from five selected secondary schools, gave informed consent and were enrolled for the study. Ages ranged between 13 and 19 years. The Child Depression Inventory (CDI) questionnaire with a cut-off point of $\geq 12$ and a semi-structured designed questionnaire to collect sociodemographic characteristic data were administered.

Results. The overall prevalence of depression among the students was $36.8 \%$. A logistic regression analysis showed students with depression had a three times higher likelihood of having a psychosocial stressor (odds ratio $(\mathrm{OR})=3.16$, confidence interval $(\mathrm{CI}) 1.96-5.10, p<0.01$ ) and ever having attempted suicide $(\mathrm{OR}=3.03, \mathrm{CI}=1.31-7.00, p<0.05)$. The prevalence of depression was significantly higher among the students who reported regular use of alcohol or other drugs (61.1\%) than among those denying such use $\left(35.7 \%, n=148 ; \chi^{2}=4.77, p=0.03\right)$. Conclusion. Over one-third of the secondary school students had depression associated with psychosocial stressors, prior suicidal attempts and perhaps adverse parenting by parents or guardians. Multilevel interventions may be necessary to prevent or reduce the rate of depression among the students.

\section{Integrating global mental health and clinical neuroscience} D Stein

Department of Psychiatry and Mental Health, University of Cape Town, South Africa

dan.stein@uct.ac.za

Global mental health has emerged as an important field that has drawn attention to the burden of mental illness and to the relative gap in mental health research and services in many regions, to framing this gap as a developmental issue, a health issue, and a human rights issue, and to developing novel approaches to building capacity, mobilising resources, and closing the research and treatment gap. Translational neuroscience has developed as an important conceptual approach to understanding the neurocircuitry and molecular basis of mental disorders, to rethinking how best to undertake research on the aetiology, assessment and treatment of these conditions, with the ultimate objective of developing entirely new approaches to prevention and intervention. There are some apparent contrasts between these fields; the first often emphasises knowledge translation, moving away from the bedside to a focus on health systems, while the latter may emphasise molecular neuroscience, focusing on transitions between bench and bedside. At the same time, there are important opportunities for integration of the two paradigms, in order to ensure that current opportunities in mental health research and services are fully maximised.

The mirror has two faces - dissociative identity disorder and the defence of pathological criminal incapacity: A South African criminal law perspective

$\underline{\text { P Stevens }}$

University of Pretoria, South Africa

philip.stevens@up.ac.za

Dissociative identity disorder poses numerous medicolegal issues whenever the insanity defence emerges. Within the context of South African (SA) criminal law, the impact of dissociative identity disorder on criminal responsibility has only been addressed very briefly in one decided case. Various questions arise as to the impact that the distinct diagnostic features of dissociative identity disorder could possibly have on the defence of pathological criminal incapacity, better known as the insanity defence, within the ambit of the SA criminal law. In this article the presenter will reflect on the mental disorder known as dissociative identity disorder or multiple personality disorder against the backdrop of the defence of pathological criminal incapacity. Reflections will be provided pertaining to the various medicolegal issues at stake whenever this defence has to be adjudicated upon.

Psychiatric features in perpetrators of homicideunsuccessful-suicide at Weskoppies Hospital in a 5-year period

P Sussman, ${ }^{1 *}$ C Kotze ${ }^{2}$

${ }^{1}$ Dr George Mukhari Academic Hospital, Ga-Rankuwa, and University of Limpopo, MEDUNSA, South Africa

${ }^{2}$ Weskoppies Psychiatric Hospital and Department of Psychiatry, University of Pretoria, South Africa

*psussman19702@hotmail.com

Background. In the absence of medical literature reporting on homicide-unsuccessful-suicide (HUS), those cases in which the perpetrator is referred for forensic psychiatric observation present an opportunity to explore psychiatric features pertaining to the event. The objective of this study is to identify possible contributing psychiatric features in HUS cases.

Method. A retrospective, single-centre, descriptive study was conducted, in which we reviewed clinical records of HUS subjects referred for observation to Weskoppies Hospital from December 2005 to January 2011. Sociodemographic and psychiatric information was reviewed. Threatened suicides without an attempt, as well as a suicide attempt more than a week after the homicide, were excluded.

Results. Nine cases were reviewed. The median age of the subjects was 29 years and seven subjects were male. Five cases involved family members. Cases involving couples demonstrated male subjects and cases involving filicide demonstrated female subjects. Only one case involved the use of a firearm. At the time of the incident, four of the cases had no psychiatric diagnosis, but did have notable interpersonal difficulties. Psychotic disorders were diagnosed in three subjects, a depressive disorder in one subject and a depressive and anxiety disorder in one subject.

Conclusion. Subjects commonly used less lethal methods than shooting. The high rate of psychiatric disorders diagnosed is in 
keeping with court referrals occurring when a mental illness is suspected. Some cases may require specialised probing before psychosis becomes apparent. Identification of psychosocial stressors and failure of coping mechanisms during periods of strife within an intimate relationship may be a focus of future research in homicide-suicide cases. Separation should possibly be investigated as an independent factor which promotes the interpersonal difficulty associated with homicide-suicide.

\section{The role of mental health review boards in the protection of mental-health-related rights in South Africa}

M Swanepoel

Department of Jurisprudence, College of Law; School of Law, University of South Africa, Pretoria; Legal practitioner for the Mental Health Review Board of Gauteng; Advocate of the High Court of South Africa swanem@unisa.ac.za

Background/Objective. We live in an increasingly complicated world and it becomes difficult to know where to seek the answers to many mental healthcare problems which affect so many of us. The objective of this presentation is to provide a brief explanation of human rights aspects with regard to mentally ill individuals and the role that the mental health review boards fulfil to maximise these practices and to ensure proper care, treatment and rehabilitation services for these individuals.

Method. A literature study of the Constitution, legislation and case law as primary sources of law is followed. In addition, textbooks and journal articles as secondary sources of law are utilised. The author also discusses practical problems that health establishments face with regard to compliance to legislation and the Review Board's role in assisting them. Results. Although much has been done in recent years to improve mental healthcare services in South Africa (SA), it is evident that a great deal more needs to be done to improve the moral standing of and to achieve social justice for the mentally ill individual. With the Constitution recognised as the supreme law in SA, the establishment of the Human Rights Commission and the enactment of the Mental Health Care Act, an infrastructure is created in SA to improve conditions with regard to mental health. The Mental Health Care Act makes provision for the appropriate care, treatment and rehabilitation of individuals who are mentally ill and to address exploitation and abuse so as to safeguard their rights. In the field of 'mental health law' decisions with consequences of the utmost gravity - decisions about compulsory medical treatment and the loss of liberty - are entrusted to mental healthcare practitioners. Yet, how do these non-legal practitioners make decisions where the legitimacy of those decisions derives from law? Mental health law examines the practical and legal terrain of duodisciplinary decision-making by, for example, addressing issues such as the legal dilemmas that mental healthcare practitioners encounter. In this regard mental health review boards play very important roles.

Conclusion. Even though individuals suffering from mental illness are among disadvantaged and vulnerable members of society, it is to be noted that rapid progress has undoubtedly been made regarding South Africa's dedication to the improvement of mental healthcare and the regulation of the mental healthcare profession. Government has promulgated extensive domestic legislation, which established the existence of mental health review boards, which have been created to ensure more supervision and accountability of care provision within health establishments and to ensure that individuals suffering from mental illness are protected during periods of vulnerability.

\section{Public mental health and mental health leadership: A South} African initiative

C Szabo

Department of Psychiatry, University of the Witwatersrand, Johannesburg, South Africa

Christopher.Szabo@wits.ac.za

Public mental health is an emerging area of focus within the discipline of psychiatry. Given that psychiatrists need an awareness of the broader context of clinical practice, public mental health would appear to be a necessary component of their knowledge base. This presentation will review aspects of public mental health, as well as describe a pilot public-private collaboration with the objective of equipping mental healthcare professionals with the requisite leadership skills to pursue equitable access to care and more effectively advocate for their patients.

Integration of South African traditional health practice with mainstream health and mental health services: The role of a psychiatrist

M Talatala

Private practice, Lesedi Private Hospital, Soweto, Johannesburg; Department of Psychiatry, University of the Witwatersrand mvuyiso@talatala.co.za

Background. Traditional medicine was originally recognised by the World Health Organization as part of the Alma Ata declaration in 1978. The African Union has recently developed a plan of action on Traditional Medicine for 2011 - 2020. In South Africa (SA), the Traditional Health Practitioners Act (THPA) was passed in 2007 to formalise the practice of African traditional medicine. The scope of traditional practice, according to the THPA, has been defined as the performance of a function, activity, process or service based on a traditional philosophy that includes the utilisation of traditional medicine or traditional practice and which has as its objective: the maintenance or restoration of physical or mental health or function; the diagnosis, treatment or prevention of a physical or mental illness; the rehabilitation of a person to enable that person to resume normal functioning within the family or community; or the physical or mental preparation of an individual for puberty, adulthood, pregnancy, childbirth and death. A 20-member Interim Traditional Health Practitioners Council was inaugurated in February 2012, with the primary role to be assisting the health ministry to achieve the integration of traditional health medicine into the national health system, within their current term of office. Traditional health practitioners are frequently consulted by mental healthcare users. At times there is a cultural interpretation of psychiatric symptoms by the mental healthcare users or their families. As a result, psychiatrists in SA often have to at least acknowledge the role of traditional health practitioners and in the near future, ways of collaborating with traditional health practitioners should be considered by psychiatrists. 


\section{ABSTRACTS}

Method. A literature search was conducted which included the review of TPHA. Discussions with psychiatrists that have an interest in the collaboration between psychiatrists and traditional health practitioners, as well as with a member of Interim Traditional Health Practitioners Council, were held.

Results. Integration of traditional health practice into mainstream medicine is still in its infancy. Collaboration between psychiatry and traditional medicine has been at best informal.

Conclusion. Psychiatrists in SA have to find ways of collaborating with traditional health practitioners. The THPA is the first step in formalising such collaboration. This presentation will reflect on the implications of this Act for mental health and psychiatric service rendering in SA.

Local NGO/government partnership addresses medication non-adherence in psychiatric outpatients: Results from a reminder and support pilot intervention

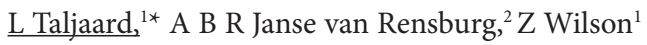

${ }^{1}$ South African Depression and Anxiety Group (SADAG)

${ }^{2}$ University of the Witwatersrand and Helen Joseph Hospital, Johannesburg, South Africa

*research@anxiety.org.za

Background. Non-adherence to medication by users of public psychiatric healthcare facilities produces more acute symptoms of mental health conditions, increases rehospitalisation, compromises treatment effectiveness, places a financial burden on healthcare costs for both institutions and individuals, and raises the risk of suicide. Reasons for non-adherence to psychiatric medication include: medication factors; illness; concurrent substance abuse or dependence; patient factors; physician factors; patient-physician factors; and social and environmental factors. The South African Depression and Anxiety Group (SADAG), in partnership with Helen Joseph Hospital, developed a Reminder and Support Adherence Programme (RSAP) as a way to remind and encourage patients to take their medication, offering them free on-going telephonic support, SMS reminders, press notifications and information. The purpose of this study was to investigate the clinic attendance and medication adherence of patients at this public sector specialist psychiatric outpatient clinic before and after a 3-month pilot implementation of the RSAP.

Method. Quantitative and qualitative methodologies, as well as retrospective and prospective components were employed. The retrospective, quantitative component included a review of participating users' demographics, clinical profile and medication adherence prior to the intervention. The prospective, qualitative component of the inquiry included a structured non-clinical pre- and post-questionnaire exploring users' knowledge and understanding of their conditions and treatment and their experiences of participating in the RSAP initiative. Descriptive statistical methods, including means and standard deviations for parametric variables, were used to analyse these variables. Ethical clearance was obtained from the University of the Witwatersrand's Human Research Ethics Committee. Results. From this total group of active outpatients $(N=607), 91$ patients (15\%) were recruited to participate. The demographic distribution of the participants indicated the majority of participants were female (76\%), black or white ( $34 \%$ and $35 \%$ respectively), with a mean age of 41.9 years.
Prior to the intervention, medication adherence was found to be $15.4 \%$. This significantly improved to $88.6 \%$. Initially, the most common reasons for medication non-adherence include: lack of reminder support (52.8\%); adverse reactions (13\%); unavailability of medication at pharmacy (11\%). Patients taking part in the RSAP also showed a markedly improved clinic appointment attendance rate (77\%), compared with non-participants (63\%), according to the Average Weighted Attendance Ratio (AWAR). An additional $10 \%$ of participants had started attending support groups during the intervention. Participants found the text reminders, ongoing lay support, information brochures and press notification to be the most helpful.

Conclusion. The SADAG RSAP was shown to improve patient medication and attendance in government facilities. During its implementation at this public referral hospital clinic, compared with patients not participating in the programme, participants also demonstrated a better attendance rate. Considering the limitations of this pilot investigation, the implementation of the SADAG RSAP in similar state sector clinics to improve adherence and attendance, can be supported.

Harmful use of alcohol and associated factors in a predominantly rural district in south-central Ethiopia: A cross-sectional community survey

$\underline{\mathrm{S} \text { Teferra, }}{ }^{1 *} \mathrm{M}$ Selamu, ${ }^{1} \mathrm{G}$ Medhin, ${ }^{2} \mathrm{~A}$ Bhana, ${ }^{3} \mathrm{C}$ Hanlon, ${ }^{1,4}$ A Fekadu ${ }^{1,5}$ ${ }^{1}$ Department of Psychiatry, College of Health Sciences, Addis Ababa University, Addis Ababa, Ethiopia

${ }^{2}$ Institute of Pathobiology, Addis Ababa University, Ethiopia

${ }^{3}$ University of KwaZulu-Natal, Durban, South Africa

${ }^{4}$ King's College London, Institute of Psychiatry, Centre for Global Mental Health, London, UK

${ }^{5}$ King's College London, Institute of Psychiatry, Centre for Affective Disorders, UK

*solomon.teferra@gmail.com

Background. Harmful use of alcohol is common in Africa and usually results in multiple physical, psychological and social problems. In this study we report prevalence and associated factors for harmful use of alcohol in a predominantly rural setting in Ethiopia. The survey was part of a multi-centre study, Programme for Improving Mental Health Care (PRIME), which is a consortium of research institutions and ministries of health of five low- and middle-income countries, namely India, Ethiopia, Nepal, South Africa and Uganda in partnership with UK institutions and the World Health Organization (WHO). The objective of PRIME is to generate evidence on approaches of integrating mental healthcare into primary care for selected priority disorders, including harmful use of alcohol. In Ethiopia PRIME is being implemented in the Sodo district, a predominantly rural district in South Ethiopia.

Method. A cross-sectional community survey was conducted involving 1500 adults, aged 18 and above, recruited using multi-stage random sampling. Data on alcohol use were collected using the Fast Alcohol Screening Test, a four-item abbreviated form of the Alcohol Use Disorder Investigation Test. Double data entry was done using EpiData software from which the data were transported to SPSS for analysis. Logistic regression was conducted to look for associations. Results. Seven hundred and forty-two (49.5\%) of the participants were men, $75 \%$ married, $42.9 \%$ non-literate, $90.7 \%$ rural residents, 
$51.6 \%$ farmers, and $54.4 \%$ considered themselves to have average relative wealth; $41.8 \%$ reported experiencing one or more life events, $41.8 \%$ reported having low social support and $26.7 \%$ of them endorsed experiencing psychological distress. The prevalence of harmful use of alcohol was $21.1 \%$. Factors associated independently with harmful alcohol use were being male, age below 60 , having experienced one or more life events and severe psychological distress.

Conclusion. High level of harmful alcohol use was detected in this mainly rural Ethiopian setting. The most productive members of the community were afflicted with this problem. Integration of services that address harmful use of alcohol and psychological distress is recommended in primary care. Public health interventions to reduce harmful use of alcohol need to be launched, coupled with the clinical service.

Psychiatric inpatients with methamphetamine psychosis in the Western Cape, South Africa: Phenomenology, risk factors, and acute treatment modalities

$\underline{\text { E Thomas }}{ }^{1 *}$ L Weich, ${ }^{1}$ C Verster, ${ }^{1}$ H Lategan, ${ }^{1} \mathrm{M} \mathrm{Kidd}^{2}$

${ }^{1}$ Department of Psychiatry, Stellenbosch University, Cape Town, South Africa

${ }^{2}$ Centre for Statistical Consultation, Stellenbosch University

*lene_tnt@live.co.za

Background. The Western Cape, South Africa, has emerged as one of the world regions with the highest levels of methamphetamine (MA) use. Because of this dramatic surge in use, methamphetamine-induced psychosis (MIP) has become the focus of increasing clinical attention. Despite this, there have been few local studies examining the clinical characteristics, demographic profile, and putative risk factors of patients admitted with MIP. Characterised as resembling schizophrenia and typically transitory in nature, identified risk factors include; heavier use, family loading of psychotic disorders, other substance use, sexual abuse, sleep deprivation and previous head-injury or neurological disorder.

Objective. To address several broad questions regarding the nature of psychotic symptoms, as well as the treatment modalities currently utilised among patients presenting with MIP.

Method. A cross-sectional survey was conducted at three level-1 Western Cape Government hospitals from 1 March 2014 to 30 August 2014. All patients, aged between 18 and 65 years, admitted to inpatient services with psychotic features associated with MA use according to Diagnostic and Statistical Manual of Mental Disorders, 5th edition (DSM-5) criteria were eligible for inclusion in this study, while a previous diagnosis of non-substance-associated psychotic disorder (e.g. schizophrenia) were exclusionary. A structured clinical interview was conducted within 1 - 7 days of admission and the Brief Psychiatric Scale (BPRS) employed as a measure of psychopathology. Results. Preliminary findings for the first 38 participants include: majority of participants were male, single, unemployed, with some secondary schooling. The median age of participants was 27 years and debut age of MA-use 21 years. While 52\% were index psychiatric admissions, $42 \%$ had previous MIP with an average of 2 previous episodes. Of the $34 \%$ participants currently prescribed an antipsychotic, $100 \%$ had defaulted treatment. The majority of participants endorsed daily MA-use by inhalation with 53\% comorbid substance use with this admission, most commonly cannabis. An average of 3 hours of sleep in the past week prior to admission was reported. The average length of stay was 7.3 days, with $50 \%$ of participants discharged home, while $50 \%$ were transferred to tertiary psychiatric care. Family history was positive in $82 \%$ for substance-use disorders and $52 \%$ for psychiatric illness; $63 \%$ of participants had a positive forensic history; $92 \%$ of these cases were reported as substance-related. Only $29 \%$ of participants had received prior rehabilitative interventions. The average BPRS total score was 69 with highest overall scores in the hallucination, hostility, unusual thought content, suspiciousness and grandiosity subscales.

Conclusion. Provisional findings point to a symptom characterisation consistent with international studies. There is a high level of concomitant substance abuse and poor adherence to existing management strategies. It is believed that the information obtained will serve to better diagnose and detect MIP susceptibility and will guide development of targeted clinical interventions and improved clinical management under these conditions.

\section{Hallucinations and similar perceptions: Conceptual clarity despite deficient definitions}

$\mathrm{W}$ van Staden

University of Pretoria, South Africa

cwvanstaden@icon.co.za

The usual definition for a hallucination is that it is a sensory awareness without a real external stimulus. The Diagnostic and Statistical Manual of Mental Disorders, 5th edition (DSM-5) defines it similarly as a key feature of psychotic disorders. This definition is problematic and in DSM-5 it is contradictory in some crucial respects. Yet it is clinically important that the definition does not mislead in distinguishing hallucinations of a psychotic kind from various other perceptions like post-traumatic flashbacks, auras, fantasies, dreams, hallucinogenic drug reactions, 'as if' perceptions, and 'pseudo-hallucinations'. This presentation will explicate the contradictions in DSM-5 regarding hallucinations and their definition by applying conceptual analysis as a particular strand of philosophy. The objective is to provide more clarity on the concepts of hallucination and related perceptions. That clarity is critically important in judging clinically whether someone is actually psychotic when hallucinating. It also has critical bearing for the validity of so-called micro-psychotic experiences in borderline personality disorder and for neuro-imagery studies of hallucinations.

Who should decide in clinical psychiatry? Facing ethical challenges of treatment non-adherence and patient idiosyncrasies

W van Staden

University of Pretoria, South Africa

cwvanstaden@icon.co.za

Does a person-centred ethic render the psychiatrist a mere puppet strung to do whatever the patient wants? Is treatment non-adherence a sign of success in person-centred health practice, in so far as it serves as an example of the patient, rather than the psychiatrist, making the choices as would seemingly be prescribed by an antipaternalistic personcentred ethic? If the answer is affirmative to either of these questions, the consequential implications are problematic if not plainly bad: the 
psychiatrist would practically have to defy his or her professional values by which the best health practice is determined and by which treatment adherence is considered a good pursuit. Yet, the practice of recognising only the patient as a person in a person-centred ethics may very well incite these conclusions and their ominous implications, even in the name of the principle in bio-ethics, 'respect for personal autonomy' Instead, this article argues a person-centred ethic should engage with the relevant values of all the persons involved in the decision-making, by which these values are accounted for in a context-specific constructive and participatory process of deliberation. Rejecting liberal individualism in this way does not amount to ethics relativism either, for practically exercising respect for diversity means that no role player needs to give up, or make equally important, his or her values. The psychiatrist may participate in this deliberation true to the professional values that determine which health practice is best, and that treatment adherence is desirable; and in turn the patient does not have to conform to these medical values and may stick to his or her values even if conflicting with medical values. Rather, as in the African process of an 'indaba', all role players may co-construct a shared decision in partnership that accounts for the common and the uncommon ground - the divergent and even conflicting values being then the best decision afforded in that situation.

\section{Advocacy for mental health: How psychiatrists and SADAG should work in partnership}

$\underline{\text { Z Wilson, }}{ }^{*}$ L Taljaard

South African Depression and Anxiety Group (SADAG), Johannesburg, South Africa

*research@anxiety.org.za

Background. It is estimated that 1 in 3 South Africans could suffer from a mental illness at any point during their lives. Despite this, treatment for mental health is still scarce or unavailable, and up to $75 \%$ of individuals do not get the treatment they require. Advocacy is a key method of raising awareness and ensuring that mental health is on the national agenda for governments and lead to improvements in policy, legislation and service development. The South African Depression and Anxiety Group (SADAG) is celebrating its 20th anniversary, and has been at the forefront of mental health patient advocacy, education and destigmatisation. It has established itself as SA's largest support network. Its expertise lies in assisting patients and callers throughout SA with mental health services.

Method. SADAG operates a national call centre with 15 toll-free helplines that assist callers suffering from depression, bipolar, panic attacks, schizophrenia, attention-deficit hyperactivity disorder, trauma, suicide, and substance abuse. SADAG has also developed and implemented an adherence programme to help patients stay on their medication and attend their clinic appointments in both the government and private settings. There has been an increased interest in suicide recently, but little research has been conducted among youth. We would like to highlight this issue with revised figures to those which Prof. Melvyn Freeman presented at the SASOP Northern sub-group Symposium. We wish to elaborate on the research SADAG does.

Results. A telephonic survey revealed that nearly all users (93.8\%) thought that the SADAG counsellors were excellent in answering their questions on the topic. We need to help patients more as psychiatrists are over-booked. Findings from a pilot-intervention programme to improve patient adherence in Helen Joseph Hospital, a public regional hospital, revealed that adherence markedly improved to $88.6 \%$. Results from our school-based suicide prevention programme evaluation indicated high levels of depression among youth, and that 1 in 5 learners have attempted suicide at least once before. We wish to highlight areas in which SADAG is working and helping to psychiatrists.

Conclusion. SADAG has for 20 years strived for promoting, valuing and respecting all individuals with mental health problems: fighting to get them into hospitals, when turned away because of no funds, encouraging them to stay on medication, running Facebook Fridays with psychiatrists twice a month, Twitter activity up to 48k per month, providing over 200 depression, panic and substance abuse support groups to help people cope again. This service outside of government hospitals does not exist. We are committed to partnerships with mental healthcare users and providers alike. Innovative primary and social care services and interventions are run every day, for hundreds of people. Our dedicated staff team, of over 100 volunteers from honours and masters students, work 12 hours a day providing massive services. We would like to tell you how you can help us and we can help your patients.

\section{POSTER PRESENTATIONS}

\author{
Stress and burnout in doctors working at a regional hospital \\ in Gauteng \\ S Avramenko, ${ }^{*}$ A Valli, G B Hope, E Libhaber, G Atilola \\ Research Methodology and Statistics, University of the Witwatersrand, \\ Johannesburg, South Africa \\ *avramenko.lana@gmail.com
}

Background/Objective. To determine the level of stress and burnout in doctors in a regional hospital in Gauteng and to find out how three different sub-scales of burnout correlate with the different types of stressors at work. The goal of the current research is to pinpoint specific stressors in order to improve the work environment.
Method. A cross-sectional study was conducted in one of the hospitals in Gauteng. Participants (doctors and nurses) received Maslach Burnout Inventory (MBI), Sources of Work Stress Inventory (SWSI), and a sociodemographic questionnaire. Data were analysed with the help of STATA 1C11.SWSI questionnaires were analysed with the help of psychologist Nikola Taylor, one of the authors of SWSI questionnaire. A regression model was applied to analyse correlation between subscales of MBI questionnaire and categories in SWSI questionnaire.

Results. From the 107 doctors working in the hospital at that time, 65 doctors returned questionnaires (response rate of 65\%). High levels of emotional exhaustion were noticeable in $52 \%$ of participating doctors; $30 \%$ had high levels of depersonalisation; and $27 \%$ experienced low 
levels of personal accomplishment. High levels of emotional exhaustion and depersonalisation were highly correlated with a lot of stressors at work. Analysis of the regression model in doctors showed that predictors of general work stress yielded a workload (positive association), that was further adjusted for role ambiguity and career advancement (all positive associations), statistically important factors. Qualitative analysis of responses from doctors highlighted problems as: understaffing, high patient/doctor ratio, difficulties with tools and equipment, problems with relationships at work with colleagues and supervisors, concerns of foreign-qualified doctors about their career prospects.

Conclusion. High levels of burnout point to a big influence of the environment, rather than personality problems. The authors feel that it is important to know what kind of stressors contribute to psychological impairment at work. It is hoped these findings will help to develop better organisation of the working environment and specific programmes in order to improve relationships at work and to reduce the level of stress, and eventually, psychological impairment from stress at work. Suggested interventions include: emotional intelligence training, team building, and time management techniques.

\section{An analysis of early developmental trauma in social anxiety disorder and post-traumatic stress disorder \\ M Bishop, ${ }^{\star}$ D Rosenstein, S Bakelaar, S Seedat \\ Stellenbosch University, Cape Town, South Africa \\ *mbishop@sun.ac.za}

Background. The early contributions of childhood trauma (emotional, physical, sexual and general) have been hypothesised to play a significant role in the development of anxiety disorders, such as posttraumatic stress disorder (PTSD) and social anxiety disorder (SAD). Objective. To assess childhood trauma differences between PTSD and SAD patients, and healthy controls; establish the frequency of events occurring in childhood, as measured by Early Trauma Inventory (ETI); the age of onset of childhood abuse and type of perpetrator; and correlate childhood traumas as measured by the ETI and Childhood Trauma Questionnaire (CTQ).

Method. We examined individuals $(N=109)$ with SAD with moderate/ severe early EDT $(n=32)$, individuals with SAD with low/no EDT $(n=29)$, individuals with PTSD with EDT $(n=17)$ and healthy controls $(n=31)$. The mean age (standard deviation) was 34 (11) years. Subjects were screened with the Mini International Neuropsychiatric Interview (MINI), Liebowitz Social Anxiety Scale (LSAS), ClinicianAdministered PTSD Scale (CAPS) and CTQ. Analysis of variance (ANOVA) was performed to assess group differences. Correlations were calculated between childhood traumas.

Results. Individuals with PTSD endorsed more physical and sexual childhood trauma while in SAD plus moderate/severe EDT emotional trauma was more common. In all groups, the occurrence of physical and emotional abuse was at $6-11$ years of age. In PTSD, the occurrence of sexual abuse was at 6 - 11 years, while sexual abuse was later (13 - 18 years) in SAD with moderate/severe EDT. For emotional abuse in all groups, the perpetrator was mostly a primary female caregiver while for sexual abuse it was a non-familial adult male. In PTSD, the perpetrator of physical abuse was mostly a male primary caregiver, compared with a female caregiver in SAD with moderate/ severe EDT. Significant positive correlations were calculated between all trauma types (CTQ and ETI). Emotional abuse and neglect had the strongest relationship with childhood trauma.

Conclusion. The contribution of childhood abuse to the development of PTSD and SAD and the differences between these groups and other anxiety disorders should not be ignored, and attention should be given to the frequency and severity of these events. The relationship of the perpetrator(s) and the age of onset of childhood abuse are also important considerations as they provide a useful starting point to assess impact over the life course. This can, in turn, guide clinicians on optimal timing for the delivery of interventions for the prevention of PTSD and SAD.

Is obsessive-compulsive disorder (OCD) with symmetry symptomatology a distinct $\mathrm{OCD}$ subtype?

E Breet ${ }_{,}^{1 *} \mathrm{C}$ Lochner, ${ }^{1} \mathrm{~N}$ McGregor, ${ }^{2} \mathrm{~S}$ Hemmings, ${ }^{2} \mathrm{~S}$ Swanevelder, ${ }^{3}$ D Stein ${ }^{1,4}$

${ }^{1}$ MRC Unit on Anxiety and Stress Disorders, Department of Psychiatry, Stellenbosch University, Cape Town, South Africa

${ }^{2}$ Department of Psychiatry, Stellenbosch University

${ }^{3}$ Biostatistics Unit, South African Medical Research Council,

Cape Town, South Africa

${ }^{4}$ Department of Psychiatry and Mental Health, University of

Cape Town, South Africa

*elsie@sun.ac.za

Background. In obsessive-compulsive disorder (OCD), symmetry concerns are common. In addition to evidence for links with several demographic and clinical features such as male gender, earlier age of onset of OCD, and comorbidity with tic disorders and obsessivecompulsive personality disorder (OCPD), symmetry symptomatology may also have specific psychobiological underpinnings. This investigation furthers previous work by examining demographic, clinical and genetic correlates of symmetry in OCD. A distinctive genotype distribution profile may provide support for OCD with symmetry symptomatology as a distinct OCD subtype.

Method. OCD patients $(N=573)$ with ages ranging from 8 to 75 years, underwent a comprehensive clinical assessment that included the Structured Clinical Interview for Diagnosis of Axis I disorders (Patient version) (SCID-I/P), and the Yale-Brown Obsessive-Compulsive Symptom Checklist and Severity Scale (Y-BOCS). DNA extracted from venous blood $(10-30 \mathrm{~mL})$ in a white subset $(n=445)$ with current symmetry concerns $(n=293(65.8 \%))$ and those without $(n=152$ (34.2\%)) was genotyped to investigate polymorphisms in dopaminergic genes previously hypothesised to be relevant to symmetry.

Results. OCD patients with symmetry obsessions and related compulsions comprised $66.1 \%$ of the total sample. Compared with those without, those with symmetry symptomatology had significantly earlier age of onset of OCD, higher OCD severity scores, longer illness duration, worse functional impairment, increased dissociation and higher rates of emotional abuse during childhood. OCD symmetry symptomatology was also significantly associated with comorbid dysthymic disorder, alcohol abuse, post-traumatic stress disorder (PSTD), bulimia and anorexia nervosa, self-injury, compulsive shopping, and obsessive-compulsive and borderline personality disorders. We found no statistically significant associations between symmetry symptomatology and COMT (rs4680), 
DRD1 (rs4532), DRD2 (rs1800497), DRD3 (rs6280), the 40bp (DAT) and 48bp (DRD4) VNTRs, and MAOA (rs1137070).

Conclusion. Our investigation of polymorphisms in dopaminergic genes does not suggest a distinct psychobiological profile for OCD patients with symmetry obsessions and related compulsions. Further work is needed to determine whether there are other (e.g. serotonergic) psychobiological mechanisms which may be responsible for symmetry concerns in OCD. Notably, our findings confirm that symmetry-related symptomatology in OCD may be associated with worse prognosis.

\section{Suicidality in OCD}

$\underline{\text { E Breet }}{ }^{1 *}$ M Kidd, ${ }^{2}$ D Stein, ${ }^{1,3}$ C Lochner ${ }^{1}$

${ }^{1}$ MRC Unit on Anxiety and Stress Disorders, Department of Psychiatry, Stellenbosch University, Cape Town, South Africa

${ }^{2}$ Centre for Statistical Consultation, Department of Statistics and Actuarial Sciences, Stellenbosch University

${ }^{3}$ Department of Psychiatry and Mental Health, University of Cape Town, South Africa

*elsie@sun.ac.za

Background. There is limited research on risk factors for suicidality in patients with obsessive-compulsive disorder (OCD).

Objective. In this study, we compared OCD patients with and without suicidality (i.e. suicide thoughts and attempts) in terms of demographic and clinical variables, including age of onset of OCD, OCD symptom dimensions and comorbid psychiatric disorders.

Method. This cross-sectional study included 543 participants with a primary diagnosis of OCD (Diagnostic and Statistical Manual of Mental Disorders, 4th edition, text revised (DSM-IV TR)). The Yale-Brown Obsessive-Compulsive Scale was used to measure OCD symptom severity, the Dimensional Yale-Brown Obsessive Compulsive Scale to assess OCD symptom dimensions, the Structural Clinical Interview for DSM-IV for Axis I disorders was used to diagnose OCD and comorbid disorders, and the SCID-OCSD was used to assess obsessivecompulsive related disorders. Participants were asked six independent questions that assessed suicide behaviour. Following preliminary analyses, $\chi^{2}$ analyses were conducted to examine whether participants with and without reported suicide behaviour differed significantly with regard to demographic and clinical variables. We employed logistical regression analyses to determine which independent variables were predictive of suicidality.

Results. Forty-four per cent of participants reported suicidal thoughts at some point in their life, while $16 \%$ reported that they had attempted suicide at least once. In the $\chi^{2}$ analyses, only major disorder, bipolar, dysthymic, panic (without agoraphobia), intermittent explosive, avoidant personality, schizotypal personality and borderline personality disorders, and kleptomania and self-injurious behaviour presented aggressive obsessions and compulsions. Logistical regression analyses demonstrated that comorbid major depressive disorder, borderline personality disorder and avoidant personality disorder remained significant predictors of suicidality.

Conclusion. Suicidality should be assessed in OCD patients with comorbid major depressive disorder, borderline personality disorder or avoidant personality disorder.

\section{Age at onset of illness in bipolar patients in South Africa}

C Grobler, ${ }^{*} \mathrm{~N}$ Smith

Walter Sisulu University, Eastern Cape, South Africa

*dr.stof@mweb.co.za

Background. Bipolar disorder (BD) is a recurrent mood disorder which typically manifests in late adolescence or early adulthood. Age at onset $(\mathrm{AAO})$ has been proposed as a variable that may distinguish more homogeneous subgroups in $\mathrm{BD}$. An early-onset $\mathrm{BD}$ has been associated with a more chronic course of the illness, comorbid substance abuse, higher comorbidity with anxiety disorders, poorer response to lithium, higher rates of suicidal behaviour and poor overall outcome.

Objective. To investigate and describe the course of illness and clinical features in a cross-section of patients with a history of mania attending public hospitals in Limpopo Province with specific attention to the $\mathrm{AAO}$ of $\mathrm{BD}$.

Method. A purposeful sample of 103 patients presenting with a history of mania between October 2009 and April 2010 to three public hospitals in the Limpopo Province were selected and interviewed using the Affective Disorder Evaluation. AAO of affective illness was analysed by dividing the groups into age brackets, namely: $<15$ years, 15 19 years, 20 - 30 years, 31 - 45 years and $>45$ years of age. Early age of onset (EAOO) in this particular study was defined as $\leq 19$ years of age. Results. The mean age of onset of first manic episode was 25 years and the mean age of onset of depression was 26 years. The majority of patients experienced their first affective episode between age 20 and 30 . The following features were identified in the EAOO group: less likely to get married ( $12.5 \%$ v. $27.85 \%)(p=0.175)$; less likely to obtain tertiary education $(12.5 \%$ v. $29.11 \%)(p=0.116)$; more likely to have a family history of BD (62.5\% v. 55.7\%) ( $p=0.641)$; less likely to attempt suicide $(20.83 \%$ v. $29.11 \%)$ in this study $(p=0.601)$; more likely to receive lithium (29.17\% v. 18.99\%) ( $p=0.393)$, zuclopenthixol $(54.17 \%$ v. $30.88 \%)(p=0.051)$ and clozapine ( $25 \%$ v. $6.33 \%)(p=0.018)$; less likely to receive oral haloperidol $(37.5 \%$ v. $53.16 \%)(p=0.244)$; more likely to have a comorbid anxiety disorder $(54.17 \%$ v. $48.1 \%)(p=0.647)$; and more likely to be rated 'markedly ill' (20.83\% v. $16.46 \%)$ to 'severely ill' $(12.5 \%$ v. $6.33 \%)$ on the CGI, which could point toward a more severe and disabling course of illness $(p=0.700)$. The only statistically significant difference that emerged from the above comparison was that the EAOO group was more likely to be prescribed clozapine $(p=0.018)$. Conclusion. Apart from the fact that clozapine was more likely to be prescribed to the EAOO group, no other statistically significant differences between early- and later-onset BD emerged in this study. Some interesting trends were observed that could be indicative of a more disabling course of illness.

Female gender and maternal mental health: Results from a study of patients presenting with mania in Limpopo Province, South Africa

C Grobler, ${ }^{*} \mathrm{~N}$ Smith

Walter Sisulu University, Eastern Cape, South Africa

*dr.stof@mweb.co.za

Background. Research with regard to bipolar disorder (BD) in South Africa (SA) remains scarce especially with regard to attention to female 
gender and maternal mental health issues in women suffering from BD. Objective. To investigate and describe the course of illness and clinical features in a cross-section of patients with a history of mania attending public hospitals in Limpopo Province with specific attention to female gender and maternal mental health with $\mathrm{BD}$ in this study population.

Method. A purposeful sample of 103 patients presenting with a history of mania between October 2009 and April 2010 to three public hospitals in the Limpopo Province were selected and interviewed using the Affective Disorder Evaluation. Specific attention was given with pertinent questions related to maternal mental health issues. Results. The majority of women in the study had their menarche at ages 15 - 16 years. Some estimates suggest that the median age of menarche worldwide is 14 but varies by geographical region, race, ethnicity and other characteristics. Twenty-six per cent reported irregular menses. Fifty-four per cent were not using contraception. Only 29\% $(n=26)$ stopped taking their medication while pregnant. Thirty per cent $(n=17)$ of women in the sample had suffered a miscarriage, the majority of whom $(n=11)$ had been prescribed valproate at some point in their illness. The rate of miscarriages is higher than the findings of a study on pregnancy outcomes in SA by Bello et al. (2010) who reported that $9.5 \%$ of pregnancies ended in spontaneous abortion and only $2.2 \%$ in stillbirths. A third of patients $(n=18)$ reported mood symptoms associated with pregnancy while $49 \%(n=28)$ reported postpartum onset of symptoms. Freeman et al. (2002) found that $67 \%$ of 50 women with $\mathrm{BD}$ experienced a postpartum mood episode.

Conclusion. This study has clearly identified several pertinent issues that are specific to women suffering from BD in SA. More than a quarter of participants experienced side-effects related to irregular menstrual cycles, with a much smaller percentage complaining of perimenstrual exacerbation of symptoms. More than half the participants used no contraception whatsoever, which is alarming in a country that has the highest number of people living with HIV/AIDS worldwide. Thirty per cent of participants had experienced a miscarriage, a rate more than triple the national average reported in other studies. During pregnancy, only a quarter of patients stopped taking their medication. The value of adequate counselling and psycho-education cannot be overemphasised in this setting, where adequate provision of information and ongoing support have the potential to prevent the unnecessary loss of pregnancies, unsafe sexual practices and side-effects of medication. Priority should be given to the issues facing these women, which would be in keeping with the Millennium Development Goals of promoting mother and child health and gender equality.

\section{Gender differences in a South African population with bipolar mood disorder \\ C Grobler, ${ }^{*}$ N Smith \\ Walter Sisulu University, Eastern Cape, South Africa \\ *dr.stof@mweb.co.za}

Background. Bipolar disorder (BD) is a serious mental disorder that affects approximately $1.3 \%$ of the population. Men and women are equally at risk of developing the illness. The clinical features and course of illness differ between men and women. The onset of BD is often later in women; they have a higher probability of experiencing mixed episodes, and are more likely to experience seasonal episodes and rapid cycling. Comorbid drug abuse is more common in men with $\mathrm{BD}$, whereas women with $\mathrm{BD}$ tend to have more comorbid eating disorders and anxiety disorders.

Objective. To investigate and describe the course of illness and clinical features in a cross-section of patients with a history of mania attending public hospitals in Limpopo Province with specific attention to the differences between males and females with BD.

Method. A purposeful sample of 103 patients presenting with a history of mania between October 2009 and April 2010 to three public hospitals in the Limpopo Province were selected and interviewed using the Affective Disorder Evaluation.

Results. Of the 103 study subjects, 45\% were male and 55\% female. Women were more likely to:

- Have a history of sexual trauma: $10 \%$ v. $0 \%(p=0.032)$.

- Be HIV-positive: $14 \%$ v. $2 \%(p=0.040)$.

- Suffer from a comorbid anxiety disorder: $63 \%$ v. $32 \%(p=0.003)$.

Men were more likely to:

- Have a forensic history: $52 \%$ v. $14 \%(p=0.001)$.

- Experience hallucinations during a manic episode: $78 \%$ v. $49 \%$ $(p=0.004)$

- Receive clozapine: $19 \%$ v. $3 \%(p=0.011)$.

- Smoke cigarettes currently: $56 \%$ v. $12 \%(p=0.001$

- Have a history of alcohol abuse $(60 \%$ v. $22 \%)(p=0.001)$ and cannabis abuse $(39 \%$ v. $2 \%)(p=0.001)$

Other interesting differences were:

- Men were less likely to be married (17\% v. $28 \%)(p=0.245)$

- There was no significant difference with regard to history of suicidal attempts $(26 \%$ v. $28 \%)(p=1.000)$; however methods used differed: men were more likely to attempt suicide by hanging $(13 \%$ v. $5 \%)(p=0.293)$; women were more like to ingest poison ( $7 \%$ v. $0 \%)$ $(p=0.126)$ or take an overdose ( $8 \%$ v. $2 \%)(p=0.221)$.

- Men were more likely to receive lithium (26\% v. $17 \%)(p=0.339)$ and clozapine ( $19 \%$ v. $3 \%)(p=0.011)$.

- Women were more likely to receive valproate $(73 \%$ v. $58 \%)$ $(p=0.141)$.

Conclusion. The findings of this study largely support prior research with regard to different expressions of BD in men and women. Of note is that women were more likely to be infected with HIV and have a history of sexual trauma, findings which are in keeping with those of the general population of South Africa, and may therefore not be specific to women with BD. Further investigation is needed to confirm these findings and determine causality and/or risk factors.

\section{Psychotropic drug prescription trends in patients presenting with mania to public hospitals in Limpopo, South Africa C Grobler, ${ }^{*} \mathrm{~N}$ Smith \\ Walter Sisulu University, Eastern Cape, South Africa \\ *dr.stof@mweb.co.za}

Background. Research is lacking with regard to the psychopharmacological management of bipolar disorder (BD) in South Africa (SA). The SA Society of Psychiatrists (SASOP) published treatment guidelines for psychiatric disorders, including BD, in 2013. Suggested treatment of $\mathrm{BD}$ is however a far cry from the reality faced in public 
service settings, especially within the rural areas of SA.

Objective. To investigate and describe the course of illness and clinical features in a cross-section of patients with a history of mania attending public hospitals in Limpopo Province with specific attention to psychotropic drugs prescribed.

Method. A purposeful sample of 103 patients presenting with a history of mania between October 2009 and April 2010 to three public hospitals in the Limpopo Province were selected and interviewed using the Affective Disorder Evaluation.

Results. At the time of the interview, the most commonly prescribed psychotropic drugs were valproate (66\%), haloperidol (50\%), zuclopenthixol decanoate (35\%), risperidone (22\%) and lithium (21\%). Forty-two per cent of subjects received orphenadrine. Only 5\% were taking an antidepressant and $5 \%$ were also on a benzodiazepine. Surprisingly few patients exhibited any extrapyramidal side-effects (EPSE) $(n=8)$ despite the fact that the majority of patients received first-generation antipsychotics (FGAs) and very few $(n=4)$ had tardive dyskinesia (TD). Valproate $(n=81)$ and lithium $(n=37)$ were the most popular choices with regard to mood stabilisers ever prescribed. Haloperidol $(n=96)$ was the most commonly ever prescribed antipsychotic drug followed by zuclopenthixol decanoate $(n=59)$. In a study by Kupfer et al. (2002), more than a third of patients with BD were taking lithium compared with approximately one-fifth $(21 \%)$ in the current study, and over $40 \%$ were taking an anticonvulsant as a mood stabiliser (v. $85.44 \%$ in the current study). More than half of the patients in the Kupfer study were taking antidepressants and $25 \%$ were taking benzodiazepines. In contrast, only $5 \%$ of patients in the current study were taking antidepressants and benzodiazepines respectively.

Conclusion. Trends in the pharmacological treatment of $\mathrm{BD}$ in this study differed markedly from trends in developed nations and also from those recently proposed by SASOP. Participants in this study were much more likely to be taking anticonvulsant medication as well as FGAs, with more than half receiving long-acting injectables. Despite the more frequent use of FGAs, relatively few EPSE and TD symptoms were noted. Study participants were less likely to be using either benzodiazepines or antidepressant medications. These differences in prescribing habits may reflect in part logistical difficulties with drug monitoring and cost constraints faced within the public health sector. The infrequent use of antidepressant medications may also be due to reluctance by clinicians to prescribe these medications because of the relatively higher rates of manic episodes seen in this study population.

\section{Substance abuse among patients with bipolar disorder in} South Africa

C Grobler, ${ }^{*}$ N Smith

Walter Sisulu University, Eastern Cape, South Africa

*dr.stof@mweb.co.za

Background. Bipolar disorder (BD) and substance-use disorders commonly occur in the same individuals. BD has a higher prevalence of substance-use disorders than any other psychiatric illness. Earlier research has indicated that individuals with both disorders have a more severe course of $\mathrm{BD}$, including earlier onset, more frequent episodes, and more complications, including anxiety- and stressrelated disorders, aggressive behaviour, legal problems, and suicide.
Objective. To investigate and describe the course of illness and clinical features in a cross-section of patients with a history of mania attending public hospitals in Limpopo Province with specific attention to the incidence and clinical characteristics of study subjects with comorbid substance abuse.

Method. A purposeful sample of 103 patients presenting with a history of mania between October 2009 and April 2010 to three public hospitals in the Limpopo Province were selected and interviewed using the Affective Disorder Evaluation.

Results. Of the 103 study subjects the following emerged with regard to current v. previous use of substances:

- The age of onset of substance use was highest in the age group 16 20 years old for nicotine, alcohol and cannabis.

- The majority started smoking cigarettes between the ages of 16 and 20 years of age and $32 \%(n=31)$ were still smoking cigarettes at the time of interview.

- Forty-one subjects (40\%) admitted to a history of abusing alcohol, with 10\% (n=10) admitting to current comorbid alcohol abuse.

- Nineteen participants (18\%) admitted to a history of cannabis abuse with $9 \%(n=9)$ currently abusing cannabis.

In the present study the following trends emerged:

- Although the rate of unemployment was high in both cannabis users and alcohol abusers, cannabis abusers were even more likely to be unemployed ( $78 \%$ v. $68 \%)(p=0.541)$.

- Cannabis abusers were less likely to receive a social grant compared with those that abused alcohol (36\% v. 53\%) $(p=0.274)$.

- Cannabis abusers were less likely than those with a history of alcohol abuse to have a comorbid anxiety disorder (26\% v. 56\%) $(p=0.051)$. This could suggest that alcohol abuse may indicate selfmedicating for anxiety.

- Those with a history of cannabis abuse were more likely to be rated as 'Severely ill' on the CGI ( $21 \%$ v. $14 \%)(p=0.711)$.

- None of the above findings were statistically significant.

Conclusion. During this study important trends emerged in participants with BD who abused substances. Although none of these differences reached statistical significance, the high rates of substance abuse in mentally ill South Africans necessitate further research into the effects of comorbid substance abuse in our population, for which these findings may serve as a starting point.

\section{Suicide attempts among bipolar patients in Limpopo Province, South Africa}

C Grobler, ${ }^{*} \mathrm{~N}$ Smith

Walter Sisulu University, Eastern Cape, South Africa

*dr.stof@mweb.co.za

Background. Suicide is known to be the leading cause of mortality in patients with bipolar disorder (BP). Several studies have suggested an association between suicidal behaviour and comorbid substance abuse, female gender and single marital status.

Objective. To investigate and describe the clinical features and course of illness in a cross-section of patients with a history of mania attending public hospitals in Limpopo Province with specific attention to those with a history of suicide attempts.

Method. A purposeful sample of 103 patients presenting with a 
history of mania between October 2009 and April 2010 to three public hospitals in the Limpopo Province were selected and interviewed using the Affective Disorder Evaluation.

Results. Twenty-seven per cent of patients in this study reported previously having attempted to commit suicide, which is much higher than $6.9 \%$ in a study from Ethiopia but similar to estimated rates of suicide of between $25 \%$ and $50 \%$ as reported by other studies. The results in this study are also in keeping with results from the EMBLEM study (a 2-year prospective, pan-European, observational study on treatment outcomes in patients with $\mathrm{BD}$ ), which reported a history of suicidal behaviour in $29.9 \%$ of study subjects. A history of suicide was associated with female gender, past alcohol or cannabis abuse and poor treatment adherence. Of those attempting suicide, hanging was the method most commonly reported, followed by overdose of medication and ingestion of poison. In a study by Schlebusch, the most frequently employed methods for completed suicide in South Africa were hanging, followed by shooting, poisoning, overdosing, gassing and burning. Conclusion. This study supports existing literature on the high rate of suicide, with more than one in four patients with BD attempting suicide during their lifetime. The methods employed during these suicide attempts are similar to those used in the general population in SA, with the exception of shooting, which may possibly reflect a lack of access to firearms by mentally ill persons because of current legislation in SA. Suicide cannot be overlooked in this patient population, and the findings provide further support for routine screening and paying particular attention to a history of suicide attempts or current suicidality in order to reduce the mortality associated with BD.

Serotonin transporter gene variants are associated with increased risk of suicide in an HIV-positive Ugandan population

$\underline{\text { S Hemmings }},{ }^{1 *}$ A Kalungi, ${ }^{2}$ S Seedat, ${ }^{1}$ M Joloba, ${ }^{3}$ E Kinyanda ${ }^{4,5}$

${ }^{1}$ Department of Psychiatry, Stellenbosch University, Cape Town, South Africa

${ }^{2}$ Department of Microbiology and Parasitology, College of Veterinary Medicine, Animal Resources and Biosecurity, Makerere University, Kampala, Uganda

${ }^{3}$ Department of Medical Microbiology, College of Health Sciences, Makerere University, Kampala, Uganda

${ }^{4}$ Department of Psychiatry, College of Health Sciences, Makerere University, Kampala, Uganda

${ }^{5}$ Medical Research Council/Uganda Virus Research Institute, Research Unit on AIDS, Entebbe, Uganda

*smjh@sun.ac.za

Background. HIV/AIDS is associated with a considerable burden of major depressive disorder (MDD). It has been found that depression is one of the foremost mental health disorders representing a barrier to antiretroviral therapy (ART) adherence, which is subsequently associated with poorer outcome. Suicide is a particular concern in the context of MDD, and MDD has been found to be highly predictive of suicidal ideation in HIV-positive individuals. In addition, lifetime suicidal ideation has been found to be increased among individuals with HIV. There is growing evidence to suggest that genes play an important role in the predisposition to suicidal behaviour, as indicated by genetic and epidemiological studies, and genes in the serotonergic system have garnered special interest in this area.

Objective. The objective of the present study was to investigate whether gene variants within the gene encoding serotonin transporter (5-HTT) were associated with suicidality in a Ugandan population with HIV/AIDS.

Methods. HIV-infected Ugandan participants $(N=569)$ were included in this study. Suicidality and comorbid psychiatric disorders were assessed using the Mini International Neuropsychiatric Interview (MINI). Risk of suicide was defined as being none-to-low, moderate or high, according to the suicidality module of the MINI. Three functional variants were investigated. An insertion-deletion polymorphism and single nucleotide polymorphism, rs25531, which are both located within the 5-HTT-linked polymorphic region (5-HTTLPR) were analysed as a triallelic polymorphism, based on results from previous publications. A variable number of tandem repeats polymorphism (VNTR) in intron 2 of the gene (STin2) was also investigated. We used mixed-effects logistic regression to investigate suicidality susceptibility. These models enabled us to control for confounders such as age and gender, as well as other sociodemographics, psychiatric comorbidity and clinical variables as fixed effects, and to correct for the variability caused by the different recruiting centres as random effects.

Results. The LA-allele was found to be significantly associated with increased risk of committing suicide $(p \leq 0.001)$. The STin2 VNTR 12-repeat allele was also found to be associated with increased risk of committing suicide $(p=0.046)$. Haplotype analysis revealed that the LA-12 allele combination was found to be significantly associated with increased risk of suicide $(p=0.002)$, compared with the SA-12 allele combination.

Conclusion. The high-5-HTT expressing alleles (LA and the STin2.12) were found to represent risk factors for increased risk of suicide in an HIV-positive Ugandan population. The results are interesting in the light of previous publications implicating 5-HTT in suicidal behaviour and suicide-related phenotypes. Understanding the risk factors, including those pertaining to biological risk, for suicidality in HIV/AIDS in the African sociocultural context is an important step towards understanding locally appropriate interventions for suicidality. Future studies should thus include gene-environment interaction analyses in order to reveal a better understanding of the role of 5-HTT in suicidality.

\section{Professional medical negligence: Is your standard of care adequate? \\ V Hitzeroth \\ Stellenbosch University, Cape Town, and private practice, Bellville, South Africa \\ psych1solway@eccsystems.co.za}

Background. Medical staff practise their profession in an ever-increasing litigious society. The practice of modern medicine and the nature of the doctor-patient relationship are continually evolving. Patients are more informed and demanding of our time and skills. Managed healthcare and treatment guidelines limit autonomous clinical decision-making. Expectations run high and the doctor's best care is often experienced as inadequate. Inevitably, patients increasingly turn to complaints and the courts to settle disputes and disagreements. When a doctor is faced 
with such a complaint or court case the standard of care offered to the patient will be critically scrutinised. Unfortunately, doctors often don't have an adequate understanding of legal concepts and processes, leaving them anxious and bewildered. One such concept is that of the standard of care that a doctor is expected to provide for his patient. Numerous and complex legal cases inform relevant jurisprudence. Legal 'to-ing and fro-ing' has opacified this concept. This presentation will attempt to clarify the legal concept of standard of care within the greater professional medical negligence field. It will provide the medical practitioner with a clearer understanding of the legal standard by which the patient's care will be judged.

Method. A detailed review of relevant legal precedents and cases as pertaining to the standard of care was undertaken. Various legal commentaries were reviewed.

Results. Legal jurisprudence regarding the accepted standard of care has evolved and developed over many decades. Much has been written, cited and published regarding an adequate standard of care. It has become impossible for a practising clinician to follow the minutiae and detailed disputes in such legal cases.

Conclusion. The evolution of the standard of care in professional medical negligence cases is presented. The background, legal precedents and recent legal complexities are introduced and discussed in order to present a clear, coherent and practical view regarding the current legal standard by which a medical professional's patient care will be judged.

An audit of failed discharges of high-frequency users at a psychiatric unit in the Eastern Cape

N Jooste, ${ }^{\star} \mathrm{C}$ Grobler, N Smith

Walter Sisulu University, Eastern Cape, South Africa

*nadia@jooste86.com

Background. Most public health systems strive to maintain mentally ill individuals in the community, as hospitalisation is costly. Psychiatric patients who are frequently readmitted to hospitals, i.e. patients with $\geq 3$ psychiatric admissions in a 2 -year period, are referred to as revolvingdoor patients. Service system variables, such as the shift to outpatient care, may influence hospital admission and it is speculated that the revolvingdoor phenomenon is the by-product of insufficient social welfare services resulting from de-institutionalisation policies, premature discharge, and inadequate preparation for discharge, rehabilitation facilities and continuity of outpatient treatment. Variables associated repeatedly with the revolving-door phenomenon are being young, single, male, substance abuse, medication non-adherence, number of prior hospitalisations and higher scores on measures of psychosis. The authors observed that there appeared to be a number of patients in their hospital that were being admitted repeatedly and a decision was taken to do an audit.

Method. This was an audit of morning hand-over round statistics at a psychiatric hospital in the Eastern Cape of failed discharges between the months of July, August and September 2013. A 'failed discharge' was defined as any patient who had been readmitted within 3 calendar months of being discharged. Once identified, case files were drawn and examined to investigate possible reasons for failed discharge. Readmission was differentiated from failed discharge and defined as any admission within 3 years of previous admission.
Results. During the period July to September 2013, 318 patients were admitted of whom $30 \%(n=95)$ were female and $70 \%(n=223)$ were male. Of these admissions, 120 (38\%) were first admissions, 173 (54\%) readmissions and 25 (8\%) failed discharges. Of the 25 failed discharges: - Sixteen were male and nine were female.

- Diagnoses were: schizophrenia $40 \%(n=10)$, substance induced psychosis $28 \%(n=7)$, psychotic disorder due to general medical condition 20\% $(n=5)$, bipolar mood disorder $8 \%(n=2)$ and schizoaffective disorder $4 \%(n=1)$.

- Evidence of substance abuse was found in $56 \%(n=14)$.

- Fourteen (56\%) stayed $<1$ month.

- Nine patients $(36 \%)$ were discharged on long-acting injections (LAIs).

- In $72 \%(n=18)$ a management plan was not discussed with a consultant.

Conclusion. The following corrective steps were taken after the audit:

- No patients to be discharged within 4 weeks without discussion with consultant.

- Discuss treatment plan with consultants.

- Psycho-educate patient and family re substances.

- Consider LAIs to improve adherence.

As an alarming $62 \%$ of all admissions in the study period were readmissions, a follow-up audit is planned to do an in-depth investigation into this group.

\section{Psychiatrists' perceptions of disputed expert witness} testimony in cases involving child custody and access

E Karim

Division of Child and Adolescent Psychiatry, Department of Psychiatry, Nelson R Mandela School of Medicine, University of KwaZulu-Natal, Durban, South Africa

Karime1@ukzn.ac.za

Background. The South African courts generally utilise an adversarial approach. Psychiatrists who are called to deliver expert witness testimony often have their opinions challenged by other expert witnesses employed by the opposing counsel.

Objective. The objective of this study was to examine what psychiatrists perceived as being reasons for disputed testimony and the factors that courts considered when deciding on whose testimony to accept.

Method. The study utilised a qualitative paradigm. The participants were all psychiatrists based in KwaZulu-Natal, who had appeared in court as expert witnesses oat least once in connection with a child custody dispute. A semi-structured interview was conducted with all participants. Results. Most KwaZulu-Natal psychiatrists indicated that they tried to avoid taking on medicolegal cases. Few study participants purposely undertook assessments on behalf of attorneys for medicolegal purposes only. Most commonly, they had become involved in court disputes in the context of delivering therapeutic services to a party to the case. Commonly, the fact that a party was receiving or had received psychiatric treatment was exploited by the opposing party in an attempt to deny or limit custody or access. Some participants indicated that they had been involved in treating the child at the focus of the dispute, and had been called to testify as to a parent's ability to handle the child's particular needs. All psychiatrists interviewed indicated that in the cases 
of disputed evidence, the opposing expert witness was a psychologist, either clinical or, in some cases, an educational psychologist. While social workers often also appeared as expert witnesses, participants rarely encountered major areas of dispute with them.

Factors mentioned by participants for disputed testimony included: - expert witnesses acting as 'hired guns'

- reluctance on the part of expert witnesses to obtain information from the treating doctor

- expert witnesses being provided with incomplete or biased information by the briefing party.

- differences in training - educational psychologist v. psychiatrist

- personal bias on the part of some expert witnesses

- perceptions of collusion between certain expert witnesses.

Less-experienced participants differed from their more-experienced colleagues in that they felt that they had not been treated fairly in court. The following factors were considered the most persuasive to the court:

- Presentation of information

- Lawyers' skill

- Expert witness status and familiarity

- Expert witness experience.

Conclusion. The study highlights real and perceived difficulties with the adversarial approach to child custody disputes. The question arises whether a more inquisitorial approach would be more appropriate.

\section{Instruments measuring emotional blunting in schizo-} phrenia: A systematic review

$\underline{\text { S Kilian, }}{ }^{*}$ L Asmal, L Philandra, A Goosen, B Chiliza, R Emsley

Department of Psychiatry, Stellenbosch University, Cape Town,

South Africa

*sanjakilian83@gmail.com

Background. Emotional blunting, also referred to as blunted affect, is frequently a prominent symptom in schizophrenia. Patients with emotional blunting have difficulty in expressing their emotions characterised by their diminished facial expression, expressive gestures and vocal expressions in reaction to emotion provoking stimuli. Emotional blunting is grouped with the negative symptoms of schizophrenia and as with other negative symptoms, it is difficult to assess and treat. The problem is that there is still a lot of uncertainty about the nature of negative symptoms. In order to address some of the questions there has been a call for researchers to study negative symptoms individually. Thus far, most research focuses on the cluster of negative symptoms.

Objective. To focus specifically on the clinical assessment of emotional blunting. More specifically the objective is to provide researchers and clinicians with an overview of the different instruments used to assess emotional blunting by focusing on the type, characteristics, administration and psychometric properties of the instruments.

Method. We searched PubMed and PsycArticles databases from July to October 2013. We selected additional studies by cross-checking article references. Two of the authors independently assessed the quality of the full-text articles using the QUADAS-2 tool. We reviewed instrument type, characteristics, psychometric properties (reliability and validity) and administration.

Results. All the instruments are easy to administer and not timeconsuming. The clinician-rated instruments take 15 - 30 minutes to administer. There is some variability with regard to the type of items included in each instrument. For example, unlike the other instruments, the Scale for Emotional Blunting also includes items measuring affect and thought content, which relates more to other negative symptoms. The clinician-rated instruments had good reliability and validity and correlated minimally with instruments measuring positive symptoms and depression. However, the selfreport instruments had poor reliability and validity.

Conclusion. Although the clinician-rated instruments have good validity and reliability we suggest that researchers and clinicians carefully consider the type of emotional blunting items included in each instrument before deciding on a particular instrument. Our findings support others who suggested that self-report instruments do not provide useful information about symptoms of emotional blunting and that more research is needed to assess patients' understanding of their symptoms.

The potential consequences of informal interpreting practices for assessment of patients in a South African psychiatric hospital

$\underline{\text { S Kilian, }}{ }^{1 *}$ L Swartz, ${ }^{2}$ T Dowling, ${ }^{3} \mathrm{M}$ Dlali, ${ }^{4} \mathrm{~B}$ Chiliza ${ }^{1}$

${ }^{1}$ Department of Psychiatry, Stellenbosch University, Cape Town, South Africa

${ }^{2}$ Alan J Flisher Centre for Public Mental Health, Department of Psychology, Stellenbosch University

${ }^{3}$ School of African Languages and Literature, University of Cape Town, South Africa

${ }^{4}$ Department of African Languages, Stellenbosch University

*sanjakilian83@gmail.com

Background. In South Africa healthcare practitioners are commonly professionals who speak only one, at most two, of their patients' languages. This provides for challenges, since many patients are not proficient in English or Afrikaans and ad hoc and haphazard arrangements are made for interpreting by untrained personnel. As part of a larger study (conducted in 2010) in a public psychiatric hospital, we report here on the potential consequences for diagnostic assessments of 13 psychiatric evaluations mediated by ad hoc interpreters who were employed as healthcare workers and household aides.

Method. The psychiatric evaluations were recorded and transcribed verbatim. The first author checked for accuracy of transcription and translations, and the two members of the author team who are both senior African language academics rechecked transcription and translation. We used the typology developed by Vasquez and Javier (1991) to study interpreter errors (i.e. omissions, additions and substitutions). All errors were independently rated by a senior psychiatrist and a senior clinical psychologist to determine whether the errors were likely to have a bearing on clinical decisions concerning the patient and to rate whether errors deemed clinically significant contributed to making the patient appear more ill psychiatrically, or less ill.

Results. Of the 57 errors recorded, $46 \%$ were rated as likely to have an impact on the goal of the clinical session. Raters concurred that the clinically significant errors contributed towards potentially making the patient look more psychiatrically ill. Detailed analyses of evaluations demonstrate the complexity of informal interpreter positioning regarding issues of diagnosis and cultural factors in illness. 


\section{ABSTRACTS}

Conclusion. Evaluations conducted where clinicians and interpreters are not trained in language and interpreting issues may create a distorted picture of the patients' mental health conditions.

A review of files to evaluate the care, treatment and legal outcomes of referrals to a South African psychiatric hospital according to the Mental Health Care Act 17 of 2002

D P Madlala, ${ }^{*}$ F Sokudela

University of Pretoria, South Africa

*dumipsm@gmail.com

Background. The Mental Health Care Act 17 of 2002 (MHCA) was introduced to combat poor care received by mentally ill persons.

Objective. To evaluate the diagnostic and treatment accuracy, as well as compliance with procedural matters related to the MHCA using a sample in the northern part of Gauteng, South Africa.

Method. Files of 200 patients admitted to Weskoppies Hospital between June and December 2009 were evaluated for admission procedures, and care, treatment and rehabilitation (CTR).

Results. From referring hospitals, 174 (87\%) persons had appropriate signs and symptoms documented in the referral note or MHCA forms; $174(87 \%)$ had appropriate diagnosis. Although about a third of individuals' treatment was not documented, more than 50\% (163) received the correct treatment. In two-thirds of patients, correction of detected abnormalities was not documented. Approximately $50 \%$ of the admissions had documents that did not adhere to MHCA provisions. At Weskoppies Hospital, CTR were considered appropriate for $92 \%$ of the persons. The legal status of the majority of patients was involuntary at discharge point. Exploration of factors associated with this is warranted. The majority of persons stayed for less than 3 months but for longer than what medical aid schemes allow in the private sector.

Conclusion. The study highlights improvements and yet some gaps in CTR given to mentally ill persons in the northern Gauteng region that might apply to the rest of the country. Medicolegal requirements stipulated by the MHCA are still a challenge a decade post-enactment but there may be a move in the right direction.

\section{Phenotypic features of patients with schizophrenia carrying de novo gene mutations}

P J Malherbe, ${ }^{*}$ J L Roos (jun), R Ehlers, M Karayiorgou, J L Roos

Department of Psychiatry, University of Pretoria, South Africa

*pjmalherbe@mweb.co.za

Background. Rare copy number variants (CNVs) and single nucleotide variants (SNVs) contribute to the aetiology of schizophrenia. In their de novo form they are highly pathogenic. This study attempts parsing schizophrenia based on the presence of such mutations. We compare patients who carry these mutations with those without detectable mutations across three categories: clinical variables, premorbid variables and course and outcome.

Method. A subset of probands was recruited from a collaborative study of schizophrenia in the Afrikaner population. Seven patients with de novo CNVs, eight with de novo SNVs and nine with no mutations were included. Follow-up evaluations were performed using the Diagnostic Interview for Genetic Studies, the Specific
Levels of Functional Assessment Scale (SLOF) and a checklist on early deviant behaviour. A mixed model of descriptive and non-descriptive statistical analysis was employed in data analysis.

Results. The lifetime diagnoses of the probands remained stable after follow-up of up to 10 years, indicating that the presence of de novo mutations does not lead to a different diagnostic course over time. The average SLOF scores showed a gradient of severity in dysfunction - worst in the CNV group and best in the no mutation group. The CNV group had significantly lower work skills, indicating worse adaptability and independence. Those with SNVs tended toward learning disability, and social dysfunction was enriched in the CNV group. In agreement with current literature we found the highest paternal age in the SNV group. Finally, clozapine use, as a marker for treatment resistance, did not differ between groups.

Conclusion. CNVs seem to predict worse functional outcome, with work skills being affected adversely. Early deviance seems to occur more frequently with SNVs. De novo mutations are not associated with differing outcomes over a 10-year follow-up and tend to occur more frequently with advanced paternal age. This study highlights valuable phenotypic characteristics in patients with schizophrenia carrying rare de novo CNVs and SNVs.

Trends of suicide in Mthatha region of South Africa over 4-year period from 2009 to 2012

B Meel $^{*}$

Walter Sisulu University, Mthatha, South Africa

•bmeel@wsu.ac.za

Background. Suicide rates often spike during economic downturns. The trends of these effects need to be studied in the Mthatha region of South Africa (SA). It is one of the poorest regions in the country.

Objective. To audit the trend of suicide in Mthatha region, SA.

Method. This is an audit of the data from the postmortem register of the Mthatha Hospital complex.

Results. There were 6405 autopsies conducted over 4 years from 2009 to 2012. Of these, 452 (7\%) people had died as a result of hanging. There is a slight decrease in the trend of hangings from 118 (26.1\%) in 2010 to $109(24.11 \%)$ in 2012 . Males (398; 88.05\%) outnumbered females (54; $11.95 \%)$. The incidence of hangings decreases with advancing age. It was highest in young adults (21 - 30-year age group: 171; (37.83\%), and lowest in those over 51 years $(4.64 \%)$. It is surprising that four $(0.88 \%)$ were children under the age of 10 years. The peak periods of these hangings are in May and November, and least in September.

Conclusion. There is a 1.4 times increasing trend of suicide among $\leq 30$-year-olds; 6.8 times higher among males in Mthatha region.

\section{Art, drama and greenery at Fort England Hospital,} Grahamstown

M Nagdee, ${ }^{1,2 \star} \mathrm{J}$ P du Toit, ${ }^{2}$ L Fike, ${ }^{2} \mathrm{~T}$ Meehan, ${ }^{3} \mathrm{~J}$ Mitchell, ${ }^{4}$ A Sutherland, ${ }^{5} \mathrm{P}$ van Tonder ${ }^{2}$

${ }^{1}$ Department of Psychology, Rhodes University, Grahamstown, South Africa

${ }^{2}$ Fort England Hospital, Grahamstown, South Africa

${ }^{3}$ Department of Psychology, Rhodes University, Grahamstown, South Africa 
${ }^{4}$ Department of Fine Arts, Rhodes University, Grahamstown, South Africa

${ }^{5}$ Department of Drama, Rhodes University, Grahamstown, South Africa *drmonagdee@gmail.com

A number of innovative projects have been introduced at Fort England Hospital (FEH) as part of the clinical and forensic rehabilitative programme. This presentation will describe the background, rationale, format and outcomes of three such projects. The objective of the FEH Art Project (commenced in 2010 in collaboration with the Departments of Psychology and Fine Arts, Rhodes University) was to use creativity in the visual arts, in a non-judgmental, non-directive environment to foster empowerment, self-expression and self-healing of participants. A selection of artwork produced has recently been exhibited at the National Arts Festival in Grahamstown. This successfully served to acknowledge and recognise the art in the public domain, and in so doing to help destigmatise mental illness, but also delivered financial rewards from sales directly to individual artists. The FEH Theatre Project (commenced in 2012 in collaboration with the Department of Drama, Rhodes University) utilises participatory drama and theatre as a means of improving self-esteem and communication skills, enhancing awareness of self and others, and facilitating personal change in the forensic mental health context. The act of theatre allows participants to imagine and rehearse positive alternatives for their own lives and futures. The Theatre Project was initiated in the National Maximum Security Unit at FEH, and has recently been extended to other state patient wards. The FEH Greenery Project (commenced in 2008), envisioned as part of a broader green agenda for mental health, is based on a growing body of evidence in support of an accessible and natural addition to existing, more conventional treatment options: ecotherapy. This approach benefits not only the physical and mental health of patient and staff participants, but also has significant aesthetic and environmental impact. The project has seen the planting of over 500 indigenous trees, and the establishment of an on-site plant nursery for sustainable propagation of suitable species. There are plans to establish a walking and hiking trail at FEH in the near future. The introduction of progressive, collaborative, multidisciplinary and evidence-based projects such as these to mental health institutions has clinically valid and significant diagnostic, therapeutic, rehabilitative and destigmatising value. They are aligned to a holistic approach to mental health recovery, while also being cost-effective in the South African context.

Correlation of severity of hypertension (systolic and diastolic blood pressures) with quality of life (QOL) of persons with essential hypertension attending outpatient clinic of University of Port Harcourt Teaching Hospital (UPTH)

$\underline{\text { A K Nkporbu }}{ }^{1 *}$ C N Stanley, ${ }^{2}$ P C Stanley ${ }^{1}$

${ }^{1}$ Department of Neuropsychiatry, University of Port Harcourt Teaching Hospital, Port Harcourt, Nigeria

${ }^{2}$ Department of Pharmaceutical Microbiology, Faculty of Pharmaceutical Sciences, University of Port Harcourt, Nigeria

*nakpigi2008@yahoo.com

Background. Essential hypertension, a non-communicable disease, is assuming an epidemic dimension, of the nature of a communicable condition in a developing nation such as Nigeria. Hypertension is a chronic disabling medication condition. The degree to which the quality of life (QOL) of the sufferers is affected is a function of its severity. Objective. To determine the relationship between the severity of hypertension and QOL of hypertensive subjects.

Method. Following ethical approval from the appropriate committee of the hospital and informed consent from the participants, 360 subjects making up the study were recruited based on the study's inclusion and exclusion criteria. This was after a pilot study. Subjects were further administered with the study's instruments including the sociodemographic and the brief version of the WHO Quality of Life instrument (WHOQOL-Bref). The sociodemographic questionnaire and WHOQOL-Bref were self-administered. The data were analysed using the SPSS version 16 statistical package. Confidence interval was set at $95 \%$ while a $p$-value of $\leq 0.05$ was considered statistically significant.

Results. The study found a higher prevalence of psychiatric comorbidity (52.2\%) among the hypertensives compared with $17.3 \%$ among people living with HIV (PLWHIV). Depressive illness had the highest prevalence in both groups: hypertension, 106 (39.4\%); PLWHIV, 47 (29.4\%). The QOL domain scores (standard deviation) for the hypertensive group were as follows: 50.97 (14.67), 55.20 (22.19), 54.51 (22.13), 50.01 (16.91) and 49.34 (22.44) for physical, psychological, social relationship, environment domains and general health facets, respectively. Also, severity of hypertension was negatively significantly correlated with QOL of persons with essential hypertension $(p<0.001)$. The only significant relationship with sociodemographic variables among the hypertensives was that physical health domain of QOL was significantly lower among the divorced and the widowed compared with other marital status groups $(p<0.001)$.

Conclusion. The findings of this study indicate that hypertension is associated with significantly lower QOL in all domains among the subjects. Furthermore, among those with hypertension, disease severity was significantly correlated with QOL. Being divorced was associated with lower QOL in some domains. The results indicate that the management of hypertension and HIV should include attention to the subjective QOL of these patients in order to enhance the quality of care.

The opinions of schizophrenia sufferers with a history of cannabis use concerning the effects of cannabis on their mental health: A cross-sectional descriptive study

$\underline{\text { R K Parshotam, }}$ * P M Joubert

Weskoppies Psychiatric Hospital, Pretoria, South Africa

*rishiekumarp@gmail.com

Background. Schizophrenia is a severe, chronic disorder with a high level of disability and a low rate of remission over the longer term. It is often comorbid with substance abuse, especially cannabis abuse. Cannabis use may not only trigger, but also perpetuate clinical features of schizophrenia in vulnerable individuals. Thus it contributes to the morbidity of schizophrenia and the burden of this mental illness in South Africa. There is little research about the opinions of schizophrenia sufferers concerning their using cannabis, and the effects of cannabis on their mental health.

Objective. To gain some clarity about the opinions of schizophrenia sufferers concerning cannabis use. 
Method. To pursue the objectives of the study 60 participants with a Diagnostic and Statistical Manual of Mental Disorders, 4th edition, text revised (DSM-IV TR) diagnosis of schizophrenia and a clinically documented history of cannabis use, were purposively selected from inpatients at Weskoppies Hospital. Having collected basic demographic data, the first researcher did face-to-face structured interviews with participants using a 16-point opinion-type questionnaire that was specifically formulated for this study. The questionnaire was formulated from the results of a previous qualitative study about schizophrenia sufferers' opinions about cannabis use.

Results. According to participants' responses $61.7 \%$ admitted to suffering from a mental illness, $95.0 \%$ admitted to using cannabis in the past, and $20.0 \%$ of participants admitted to the current use of cannabis. Regarding the effect of cannabis on participants' mental illness, 51.7\% responded that cannabis had adverse effects on their mental health, $26.7 \%$ that their mental illness was caused by using an illicit drug other than cannabis, $26.7 \%$ that only impure cannabis has adverse effects on their mental health, and $48.3 \%$ that only using too much cannabis has bad effects on their mind. Regarding possible positive effects of cannabis, participants' responses were as follows: $58.3 \%$ responded that cannabis use helped to reduce their tension, $56.7 \%$ that cannabis use helped to reduce their anxiety, $66.7 \%$ that cannabis use helped to lift their mood, $63.3 \%$ that cannabis use helped them to relax, $60.0 \%$ that cannabis use helped to relieve their boredom, $43.3 \%$ that cannabis use helped to make them feel more energetic, 58.3\% that cannabis use helped them to sleep better, $13.3 \%$ that cannabis use helped to reduce auditory hallucinations, and $31.7 \%$ that the good effects of cannabis outweighed the negative effects of cannabis.

Conclusion. Despite this group of participants responding that they used less cannabis in the present than in the past, in general they had many positive views of the effects of cannabis on their mental health. That is so despite just more than half of them responding that cannabis may have adverse effects on their mental health.

Social anxiety disorder in the context of early developmental trauma: Structural magnetic resonance imaging (sMRI) and proton magnetic resonance imaging findings (1H-MRS)

D Rosenstein ${ }^{1 \star}$ AT Hess, ${ }^{2}$ F Ahmed-Leitao, ${ }^{1} \mathrm{~J}$ Zwart, ${ }^{3}$ E Meintjies, ${ }^{4}$ S Seedat ${ }^{1}$

${ }^{1}$ South African Research Chairs Initiative in Post-traumatic Stress Disorder, Department of Psychiatry, Stellenbosch University, Cape Town, South Africa

${ }^{2}$ University of Oxford Centre for Clinical Magnetic Resonance Research, UK

${ }^{3}$ Department of Physics and Astronomy, University of the Western Cape, Bellville, South Africa

${ }^{4}$ Biomedical Engineering, University of Cape Town, South Africa

*rosensteind@gmail.com

Background. Individuals with anxiety disorders demonstrate alterations in the neurometabolism of the limbic system, specifically the amygdaloid complex. This has also been demonstrated in social anxiety disorder (SAD). Early developmental trauma (EDT) has been hypothesised to play a significant role in the pathophysiology of SAD; however, there have been no published proton magnetic resonance spectroscopy (1H-MRS) or sMRI studies in SAD in the context of EDT. We compared individuals with SAD $(n=26)$ with EDT with individuals with SAD without EDT $(n=20)$ and healthy controls $(n=22)$.

Method. Subjects were screened with the Mini International Neuropsychiatric Interview, Liebowitz Social Anxiety Scale and Childhood Trauma Questionnaire. Scanning was carried out on a 3T Siemens Allegra scanner. $1 \mathrm{H}-\mathrm{MRS}$ and sMRI was used to elucidate neurometabolite differences and structural differences respectively. Bayesian and null hypothesis significance testing (NHST) was performed to compute differences in probabilities of all neurometabolites between the groups.

Results. Probable differences were found in Ins (JZS BF=0.008294412; BEST effect size $1.2195 \%$ HDI $(0.496,1.96))$, PCr (JZS BF $=0.3306585$; BEST effect size -0.808 , 95\% HDI $(-0.135,-1.51)$ ), NAA (JZS $\mathrm{BF}=0.1555764$; BEST effect size $-0.0422,95 \%$ HDI $(-1.52,-0.165))$ and Glx (BEST effect size 0.768, 95\% HDI $(1.48,0.0861)$ ) in the left amygdala in the SAD with EDT group and probable differences were found in NAA ((BEST effect size -0.0422 , 95\% HDI $(-1.52,-0.165)$ ), Gln (JZS BF $=0.3068417$, BEST effect size $0.579,95 \%$ HDI $(0.112,1.52))$ and Glx (BEST effect size $0.768,95 \%$ HDI $(1.48,0.0861)$ ) in the SAD without EDT group. Statistically significant differences were found in PCR $(M=0.83, S D=0.34)$ between the SAD with EDT and SAD without EDT groups and NAA $(\mathrm{M}=-0.92, \mathrm{SD}=0.31)$ and $\mathrm{Ins}(\mathrm{M}=1.88, \mathrm{SD}=0.43)$ in the SAD with EDT group. Statistically significant differences were found in $\mathrm{Gln}(\mathrm{M}=0.83, \mathrm{SD}=0.34)$ and NAA $(\mathrm{M}=0.77, \mathrm{SD}=0.31)$ in the SAD without EDT group. No differences were found in amygdala volume. We found convergence between NHST and Bayesian analyses, with greater sensitivity in Bayesian analysis. Group differences in sMRI findings were found in the left and right anterior cingulate cortex, and left thalamus in the SAD with EDT group, and the left caudate nucleus between the SAD with EDT and SAD without EDT group.

Discussion. These findings contrast with previous research that has examined alterations neurometabolites in limbic structures in SAD. The structural differences are similar to research findings in anxiety disorders and what has been found in some studies of SAD, OCD and EDT. EDT plays a specific role in the pathophysiology of SAD, as evidenced by a difference in a number of brain circuits in individuals with SAD and EDT compared with individuals with SAD without EDT.

\section{Professionals' and patients' perspectives towards disclosing} incidental findings of pleiotropic results

N Schuitmaker, ${ }^{*}$ S Seedat

Stellenbosch University, Cape Town, South Africa

*nicoles@sun.ac.za

Background. Incidental findings (IFs) can arise in many medical contexts, but they are most frequently found in imaging, oncological and genetic studies. Genetics research, specifically pleiotropic research, inherently potentiates IFs, leaving researchers and clinicians in need of direction regarding the disclosure of such information. Many factors play a role in making such a decision, i.e. ethical factors, practical and technical factors, and the logistics of disclosure. Official guidelines pertaining to this matter have not been established in South Africa and more research is needed to aid this process. It would 
be of value to have a greater understanding of people's perspectives regarding the disclosure of IFs to patients in the context of pleiotropic results.

Objective. To investigate the different opinions that researchers, students, and research participants have towards IFs in genetics research.

Method. The sample will consist of approximately 300 participants, specifically researchers and students from the Faculties of Medicine and Health Sciences at Stellenbosch University and the University of Cape Town. Research participants from studies being conducted at the Stellenbosch University Psychiatry Department will also be approached to participate. A cross-sectional online survey will be sent to potential participants. Those that complete the survey will be asked whether they are willing to participate in a telephonic semi-structured interview.

Results. We hypothesise that the majority of participants will be supportive of the disclosure of accurate and clinically actionable IFs. However, there will be mixed opinions between researchers, students, and patients about whether to disclose IFs that are not necessarily clinically actionable/significant. There will also be mixed views about the importance of the different factors influencing the return of IFs.

Conclusion. Clear yet flexible guidelines that assist researchers to effectively return IFs need to be developed. Further research is necessary in order to develop these procedures.

\section{Associations between brain-derived neurotrophic factor plasma levels, cognition and later post-traumatic stress disorder \\ $\underline{\text { S Suliman }},{ }^{1 *}$ D J Stein, ${ }^{2}$ S Seedat ${ }^{1}$ \\ ${ }^{1}$ Department of Psychiatry, Stellenbosch University, Cape Town, \\ South Africa \\ ${ }^{2}$ MRC Anxiety Disorders Unit, Department of Psychiatry, Stellenbosch \\ University and Department of Psychiatry and Mental Health, University of Cape Town, Cape Town, South Africa \\ *sharain@sun.ac.za}

Background. Brain-derived neurotrophic factor (BDNF) is a neurotrophin (NT) which fosters the growth, survival and differentiation of neurons in the peripheral and central nervous systems. BDNF is also present in the bloodstream and derives from different sources, including platelets and the brain. Although there are some data indicating that BDNF can be considered a marker of stress-related or mood disorders, the role of BDNF in post-traumatic stress disorder (PTSD) is currently still unclear, with some studies finding higher levels, some lower levels and yet others no difference in those with the disorder. Additionally, it is not known whether low plasma BDNF levels in acute trauma represent a state marker for PTSD. Further, BDNF has been shown to mediate synaptic plasticity, which underlies hippocampus-related functions, such as learning and memory, functions that are frequently impaired in individuals with PTSD.

Objectives. To determine whether BDNF levels early after trauma are associated with cognitive functioning and predictive of PTSD severity. Method. We assessed plasma BDNF levels and cognitive functions in 120 adult motor-vehicle accident survivors (mean (standard deviation) age: 33.05 (10.54) years) within 2 weeks of the accident and assessed for PTSD 3 and 6 months later. We conducted correlations between BDNF plasma levels and (i) cognitive functions (learning, visual and verbal memory, executive functions and information processing); (ii) PTSD severity 3 and 6 months after the trauma.

Results. We did not find an association between BDNF plasma levels and PTSD severity. BDNF levels were however negatively correlated with a test of executive functioning (Tower of London), so that poorer planning correlated with lower BDNF levels.

Conclusion. This association of lower BDNF levels with poorer executive function has previously been reported, possibly reflecting the role of BDNF in hippocampal function. Given that lower BDNF levels might be associated with reductions in hippocampal volumes and cognitive disturbances associated with PTSD, and that treatment has been found to be effective in increasing BDNF levels, BDNF is a potential biomarker of PTSD, in diagnosis, disease progression and pharmacotherapy response. It would, however, be useful to clarify the relationship further. Further evidence, ideally from larger prospective studies that take a variety of clinical, demographic and lifestyle factors into account, is needed.

\section{Predictors of post-traumatic stress disorder}

$\underline{\text { S Suliman }}{ }^{1 *}$ D J Stein, ${ }^{2}$ S Seedat ${ }^{1}$

${ }^{1}$ Department of Psychiatry, Stellenbosch University, Cape Town, South Africa

${ }^{2}$ MRC Anxiety Disorders Unit, Department of Psychiatry, Stellenbosch University and Department of Psychiatry and Mental Health, University of Cape Town, Cape Town, South Africa

*sharain@sun.ac.za

Background. Although acute responses to traumatic stress generally resolve within a few weeks, some individuals experience severe and persistent problems, such as post-traumatic stress disorder (PTSD). While studies have identified a variety of predictors of PTSD, not all data are consistent. This longitudinal study examined the predictive power of neurocognitive deficits and psychiatric symptom variables with regard to PTSD severity.

Method. A total of 131 adult road traffic collision (RTC) survivors were assessed within 2 weeks of the RTC, including social, demographic, lifestyle, clinical and neuropsychological measures. Participants were then followed up 3 and 6 months later to determine severity of PTSD.

Results. Impairment on tests of information processing, executive functioning, verbal learning and motor speed predicted PTSD severity when neuropsychological, psychiatric symptoms and sociodemographic factors were taken into account. Clinical variables (initial symptoms, psychiatric diagnoses, disability, trait anxiety, perceived stress, negative cognitions, sleep) were associated with 3- and 6-month PTSD severity, but only trait anxiety was predictive of PTSD severity. Ethnicity and education were also found to be predictive.

Conclusion. These findings suggest implementation of a holistic approach to screening for PTSD and support a need for interventions that target neurocognitive, clinical and social variables. Early, targeted profiling of this group of trauma survivors can inform early clinical interventions and policy.

Current practice of psychiatry in private practice in South Africa should contribute to the research agenda and the teaching of psychiatry

M Talatala 
Private practice, Lesedi Private Hospital, Soweto, Johannesburg; Department of Psychiatry, University of the Witwatersrand, Johannesburg

mvuyiso@talatala.co.za

Background. Almost 50\% if not more of psychiatrists in South Africa (SA) are in private practice. A large number of mental healthcare users are treated in private health facilities. There is a growing number of private psychiatry inpatient facilities in SA and in provinces like Gauteng these offer involuntary care, as well as child and adolescent care. There are protocols and practices in private practice that are deemed essential and yet they may not have been thoroughly researched in SA and at times psychiatrists may not have been trained adequately for these. The practice of group therapy by psychiatrists and the ongoing negotiations on practice of psychiatry that will allow for alternative reimbursement models are examples of ongoing activity that should be researched and taught even in SA academic facilities. The introduction of National Health Insurance will require some level of harmonisation of what is practised by private psychiatrists with what is currently being practised in public facilities.

Method. Literature review and review of relevant documents in the archives of the Psychiatry Management Group, a management company of psychiatrists in private practice, was conducted.

Results. Progress is being made on health interventions in private practice in SA and these may make a contribution in the envisaged National Health Insurance (NHI). However, there is no clear collaboration and harmonisation between private and public psychiatry practice in SA.

Conclusion. Collaboration in teaching, practice and research of psychiatry between the private psychiatry sector and state psychiatry sector is essential in improving the provision of psychiatry in SA.

\section{A comparative study of the clinical characteristics and symptom profile in outpatients diagnosed with methamphetamine-induced psychotic disorder v. schizophrenia \\ H Temmingh, ${ }^{*}$ J Hsieh, D J Stein, F Howells \\ Department of Psychiatry and Mental Health, University of Cape Town, South Africa \\ *henk.temmingh@uct.ac.za}

Background. In the acute phase of illness the clinical symptom profile of methamphetamine-induced psychotic disorder (MIPD) closely resembles that of schizophrenia, often making it very difficult to distinguish these disorders. Studies among inpatients have shown that there are no differences in the psychotic symptom profile between these disorders. In turn, like patients with MIPD, as many as $75 \%$ of patients with a diagnosis of schizophrenia have comorbid substance use disorders (dual diagnosis). Our objective was to compare the clinical characteristics and symptoms in a group of patients with a diagnosis of methamphetamine-induced psychosis to a group of patients with schizophrenia.

Method. We recruited patients from community outpatient departments. We determined diagnosis using the SCID-I-RV. The Positive and Negative Syndrome Scale (PANSS) was used to determine symptom structure, Global Assessment of Functioning (GAF) scores to measure functional impairment, and the Alcohol, Smoking and Substance Involvement Screening Test (ASSIST) to measure substance-use severity. Results. The total sample consisted of 61 patients, 27 with a diagnosis of MIPD and 34 with schizophrenia. There were no differences in the gender or educational level between the two groups. MIPD patients were significantly younger $(p=0.002)$ compared with schizophrenia patients. In turn the median time since first diagnosis for the schizophrenia group was significantly longer (66 v.12 months, $p<0.001)$. Although total and negative PANSS scores did not differ significantly between the two groups, patients with schizophrenia had significantly higher scores on the PANSS positive scale $(p=0.012)$. In turn, ASSIST total substance involvement score (TSI-score) was significantly higher in the MIPD compared with the schizophrenia group ( $p<0.001)$, as were the specific substance involvement scores for alcohol, cannabis, methamphetamine and methaqualone. There was a positive correlation between PANSS positive score and TSI-scores in the MIPD group that was significant at the $10 \%$ level (Spearman's $\rho=0.33, p=0.088$ ); whereas this association was much weaker in the schizophrenia group (Spearman's $\rho=0.14, p=0.408$ ). We found no significant differences in the GAF score between the two groups.

Conclusion. Over time, schizophrenia and MIPD psychosis can be differentiated, perhaps more so in groups with prominent residual positive symptoms and treatment resistance. Both groups showed impairment in functioning. It remains unclear whether MIPD represents an early at-risk state for the development of schizophrenia. Limitations include the cross-sectional nature of data and small sample size.

Extrapyramidal motor side-effects in antipsychotic-treated patients with non-affective psychosis and major affective disorders with and without co-occurring alcohol, cannabis and methamphetamine use disorders

$\underline{\mathrm{H} \text { Temmingh, }}{ }^{1 *}$ B Broekhof, ${ }^{2}$ G Sibeko, ${ }^{3}$ D J Stein ${ }^{1}$

${ }^{1}$ Department of Psychiatry and Mental Health University of

Cape Town, South Africa

${ }^{2}$ University of Amsterdam, The Netherlands

${ }^{3}$ University of Cape Town

^henk.temmingh@uct.ac.za

Background. Comorbid substance use disorders occur in as many as $75 \%$ of people with schizophrenia and bipolar disorder. Alcohol, cannabis and cocaine have been shown to lead to anatomical and physiological changes in basal ganglia structures, including striatal dopaminergic down-regulation and the induction of extrapyramidal motor side-effects (EPSE). Whereas the overall effect is likely to be small, the associations with EPSE are the strongest for cocaine, weakest for alcohol use, and remain unclear for cannabis use, with many studies not specifying substance-use type. Little is known about the impact of methamphetamine on the risk of EPSE in patients with schizophrenia and other disorders such as bipolar disorder for which antipsychotics are indicated.

Objective. Our objective was determine if there is an association between alcohol, cannabis and methamphetamine use disorders and the presence of ESPE in patients prescribed antipsychotics.

Method. We determined diagnosis using the SCID-I for the Diagnostic 
and Statistical Manual of Mental Disorders, 4th edition, text revised (DSM-IV-TR) and the presence and severity of substance use disorders using module $\mathrm{E}$ of the SCID-I. We determined the presence of akathisia using the Barnes Akathisia Rating Scale (BARS), parkinsonism using the Simpson-Angus Scale (SAS) and tardive dyskinesia using the Abnormal Involuntary Movement Scale (AIMS). We constructed a series of logistic regression models. The primary outcome was the presence of any EPSEs reaching operationalised threshold on the different EPSE scales.

Results. The total sample consisted of 96 patients. The most common diagnosis was schizophrenia (55.2\%), followed by bipolar disorder (20.8\%); schizoaffective disorder (11.4\%) and substanceinduced psychotic disorder (12.5\%). Alcohol-use disorders occurred in $30.2 \%$, cannabis-use disorders in $39.6 \%$ and methamphetamineuse disorders in $25 \%$ of participants. As many as $41.7 \%$ had at least one EPSE, with $38.5 \%$ having parkinsonism, 10.4\% akathisia, and $1.04 \%$ narrowly defined tardive dyskinesia while $9.4 \%$ had broadly defined dyskinesia. Participants with EPSE were more likely to be male. Multivariate logistic regression models adjusted for age, gender, diagnosis and treatment with first-generation antipsychotics revealed no association between alcohol- or cannabis-use disorders and EPSEs. Patients with a methamphetamine dependence were more likely to have EPSEs (odds ratio $(\mathrm{OR})=3.23$ ) and this association approached significance at the $5 \%$ level $(p=0.051,95 \%$ confidence interval $(\mathrm{CI})=0.99-10.5)$. When looking at patients with only methamphetamine abuse this association remained non-significant $(p=0.824)$. Sensitivity analysis with a definition of broadly defined dyskinesia on the AIMS resulted in a significant association between methamphetamine dependence and EPSE $(\mathrm{OR}=3.87, p=0.030,95 \% \mathrm{CI}=1.13$ - 13.1).

Conclusion. Patients with more severe methamphetamine use disorders (i.e. dependence) may have more EPSEs when treated with antipsychotics. Clinicians should assess substance use severity and monitor side-effects on a regular basis in dual diagnosis patients. Limited conclusions can be drawn on causality because of the crosssectional nature of the study. Other limitations include no adjustment for antipsychotic dose.

Sniffing out the olfactory reference syndrome: A case report E Thomas, ${ }^{1 \star}$ B Chiliza, ${ }^{1}$ C Lochner, ${ }^{2}$ D Stein ${ }^{2,3}$

${ }^{1}$ Department of Psychiatry, Stellenbosch University, Cape Town, South Africa

${ }^{2}$ MRC Unit on Anxiety and Stress Disorders, Department of Psychiatry, Stellenbosch University, South Africa

${ }^{3}$ Department of Psychiatry and Mental Health, University of Cape Town, South Africa

*lene_tnt@live.co.za

Background. Olfactory reference syndrome (ORS) is characterised by the erroneous belief that one emits an unpleasant body odour. This results in significant distress and is often accompanied by repetitive behaviour such as frequent showering in an attempt to camouflage the perceived odour. The body odour concerns may have a delusional quality and do not respond to simple reassurance or counter-example. We report a case of an ORS patient who had undergone polysurgery and presented with depressive symptomatology.
Case report. A 46-year-old single male referred to Consultation Liaison Psychiatry assessment with a complaint of a bad odour that had been emanating from his body for the past 8 years. Relief had been sought from several medical specialists, and surgery including tubinoplasty, and a tonsillectomy were performed, yet the odour and the resulting distress persisted. The Brown Assessment of Beliefs Scale (BABS) was 20, correlating with a poor degree of insight. The Beck Depressive Inventory II (BDI-II) score was high at 30. Fluoxetine was commenced owing to marked comorbid depressive symptomatology. Weekly cognitive behavioural therapy commenced in a collaborative, non-didactic and graded fashion.

Results. At 3-month follow-up he had responded well to a dose of $60 \mathrm{mg}$ fluoxetine, with lessened depressive symptomatology as well as time spent engaging in safety behaviours. Delusional beliefs and preoccupations however remained highly salient, and risperidone was initiated and titrated to response. At 5-month follow-up depressive symptoms had abated (BDI=4) with reported improvement in functioning. Although the referential thoughts still continued, they were much less frequent and intense and he was able to express doubt regarding the veracity of his perceptions (BABS=13).

Conclusion. ORS may lead to significant disability, yet often goes unrecognised for many years. For many patients poor insight will contribute to their reluctance to consider psychiatric treatment. The literature regarding ORS treatment remains limited. Whether adding a cognitive behavioural model in combination with pharmacotherapy results in improved outcomes has not been systematically studied. However, it is clear that improvement in ORS can take place with all modalities of treatment. In this case it was demonstrated that a multimodal treatment approach comprising judicious medication use, combined with cognitive behavioural therapy in the context of a therapeutic alliance, yielded therapeutic success.

\section{BDNF Val66Met polymorphism and plasma levels in acutely traumatised road traffic accident survivors}

$\underline{L}$ van den Heuvel, ${ }^{*}$ S Suliman, S Hemmings, S Seedat

Department of Psychiatry, Stellenbosch University, Cape Town, South Africa

*luellaz@gmail.com; llvdh@sun.ac.za

Method. One hundred and fifteen adult participants (mean (standard deviation) age 33.18 (10.60); gender $58.3 \%$ male) who survived a road traffic accident (RTA) were recruited from four hospitals in Cape Town. Clinical and laboratory assessments were performed within 10.01 (4.86) days of RTA exposure. Acute stress disorder (ASD) was diagnosed in 47 (42.3\%) participants based on a cut-off of 56 on the ASD Scale (ASDS). Digit span backwards (5.25 (2.29)) was used to assess working memory performance. ELISA was used to measure early morning brain-derived neurotrophic factor (BDNF) plasma levels and Val66Met genotyping was performed.

Results. Eighty-four participants (68.3\%) were BDNF Val66Val homozygous; 28 (22.8\%) were heterozygous and 3 (2.4\%) were BDNF Met66Met homozygous. Met carrier groups were combined for analysis. BDNF plasma levels (51.27 (41.63)) were not significantly associated $(p \leq 0.05)$ with BDNF Val66Met genotype. Neither BDNF Val66Met genotype nor plasma BDNF was significantly $(p \leq 0.05)$ associated with 
the presence or severity of ASD or working memory impairments. Conclusion. In acutely traumatised RTA survivors we found no association between BDNF Val66Met genotype, BDNF plasma level and ASD or working memory impairments. In acutely traumatised RTA survivors many factors can influence BDNF plasma levels, such as tissue injury, diet, medication and substance use and therefore many possible confounders exist. Individuals were assessed within 2 weeks of RTA exposure and therefore the presence of ASD was being assessed only in the acute period following trauma exposure. A relationship between BDNF Val66Met, BDNF plasma level, PTSD and working memory may be more reliably detected if measures are repeated and followed up longitudinally.

Patients' perceived precipitating adverse life events of a current major depressive episode: A comparison between bipolar disorder and major depressive disorder

$\underline{\mathrm{R} A \text { van Schoor, }}{ }^{*} \mathrm{P} \mathrm{M}$ Joubert

Department of Psychiatry, University of Pretoria, South Africa

*robynannevs@gmail.com

Background. The study examines what participants suffering from major depressive disorder (MDD) or bipolar disorder (BD) perceive as the precipitating adverse life event of their current major depressive episode (MDE). The severity and types of adverse life events were compared between participants suffering from MDD and BD.

Method. Consenting, adult in- and outpatients were sourced from Weskoppies Hospital, Steve Biko Academic Hospital, Tshwane district hospital, Denmar psychiatric hospital and Vista Clinic in the Pretoria area. A semi-structured questionnaire was used to gain information including: demographic data, diagnosis and the course of the disorder (including the number of previous MDEs and the age at which the first MDE occurred). The perceived precipitating adverse life event was detailed for each participant and a severity value named a life change unit (LCU) score based on the recent life changes questionnaire (Miller and Rahe, 1997) was assigned to each participant's perceived precipitant.

Results. A total of $12.7 \%$ of participants were experiencing their first MDE. In those participants who had had prior episodes, the average number (standard deviation) of previous episodes was 3.86 (2.46). The mean approximate age at first onset of a MDE was 24.81 (10.9) years. The BD group had significantly more previous MDEs than the MDD group.

Although the average LCU scores were higher in the BD group than the MDD group, this did not reach statistical significance. This disconfirms the null hypothesis that there may be a difference in the severity of the perceived precipitant between the two groups of patients based on clinical diagnosis. The LCU scores were analysed within subcategories of types of psychosocial stressors. In this sample of patients, individuals with $\mathrm{BD}$ experienced significantly more problems associated with the workplace as precipitants of a current MDE than individuals with MDD.

Conclusion. The vast majority of participants could link an adverse life event to the onset of their current MDE. The study did not find a differential response to adverse life events when patients with $\mathrm{BD}$ and patients with MDD were compared. The severity of the social precipitants did not seem to differ between the two groups based on the clinical diagnosis. The notion of a 'kindling effect' is not supported by the outcome of this study. Because some study participants experienced adverse life events not accounted for by the Holmes and Rahe scale, other scales should be considered for similar studies.

\section{Does religious identification of South African psychiatrists matter in their approach to religious matters in clinical practice?}

M Welgemoed, ${ }^{*} \mathrm{C}$ W van Staden

Department of Psychiatry, University of Pretoria and Weskoppies Psychiatric Hospital, Pretoria, South Africa

*mariuswelgemoed@gmail.com

Background. It is not known whether psychiatrists' approach to religious matters in clinical practice reflects their own identification or nonidentification with religion or their being active in religious activities. This question was investigated among South African (SA) psychiatrists and psychiatry registrars including the importance they attach to the religious beliefs of patients for diagnostic and therapeutic purposes.

Method. Respondents from the SA Society of Psychiatrists (SASOP) completed a purpose-designed questionnaire anonymously online. Respondents were compared statistically according to whether they identified with a religion and the regularity of their participation in religious activities. Further comparisons were made based on gender, and years of clinical experience.

Results. Participants who identified with a religion showed no statistical differences in comparison with those who did not, regarding how they view the importance of a patient's religious beliefs for purposes of diagnosis, general management, psychotherapy, pharmacotherapy, recovery from acute episode, maintenance of recovery or remission, time to be spent on religious education, referral for religious/spiritual counselling according to patient's own beliefs; referral when patient and participant are of different religions; and whether referral is considered harmful when a patient's religious beliefs are similar to or different from the participant's. Statistical differences were found where participants who did not identify with a religion were more likely to indicate religion has 'little importance' for the purpose of understanding the patient; and were more likely to indicate 'no' when asked if they would refer a patient for religious/spiritual counselling. When comparing regularity of participation in religious gatherings, participants who indicated their participation as 'no/never' were more likely to answer 'no' when asked if they would refer a patient for religious/ spiritual counselling, even when of similar religion to that of their patient. In comparing gender, males were more likely to answer 'yes' than females when asked if they consider religious/spiritual counselling (in accordance with the patient's own religious beliefs) potentially harmful when the patient's religion is different from the participant's.

Conclusion. It appears that SA psychiatrists' identification with religion and regularity of participation in religious gatherings do not influence their approach to religious matters of their patients in most respects. The exception seems to be those psychiatrists who do not identify with a religion (about 16\%) who tend to say they do not refer for religious counselling, and consider the patient's religious identification to be of little importance in understanding the patient. 